\title{
Mobility evaluation of wheeled robots on soft terrain: Effect of internal force distribution
}

\author{
Bahareh Ghotbi, Francisco González, József Kövecses, Jorge Angeles
}

This is a post-peer-review, pre-copyedit version of an article published in Mechanism and Machine Theory. The final authenticated version is available online at: https://doi.org/10.1016/j.mechmachtheory . 2016.02 .005 .

This document is licensed under a CC-BY-NC-ND license.

\begin{abstract}
Many applications of wheeled robots include operations in unstructured environments. Optimizing vehicle mobility is of key importance in these cases. Reduced mobility can limit the ability of the robot to achieve the mission goals and can even render it immobile in extreme cases. In this paper, some aspects of the effect of the wheel-ground interaction force distribution on mobility are investigated. A performance index based on the normal force distribution is used to compare different design layouts and vehicle configurations. The validity of this index was assessed using both multibody dynamics simulation and experimental results obtained with a six-wheeled rover prototype. Results confirmed that modifying the system configuration and employing active suspensions to alter the normal force distribution can lead to an increase of traction force available at the wheel-terrain interfaces, thus improving rover mobility. Finally, the study was extended to consider the change of soil properties during operation due to the multipass effect. Optimum load distributions were obtained as the solution of a constrained maximization problem.
\end{abstract}

Keywords: Wheeled mobile robots, Soft terrain, Load distribution, Multipass effect 


\section{Introduction}

Optimizing vehicle mobility is an important goal in the design and operation of wheeled robots on soft soil. Degraded wheel-terrain interaction conditions can result in the vehicle not being able to develop the required traction to complete certain maneuvers. This may result in failure to achieve the mission goals and in some cases the loss of the robot. In spite of its importance, no general agreement exists in the literature about the precise meaning of mobility in the context of operations on soft terrain. The concept has a precise meaning for wheeled robots moving on rigid, flat ground [8], when it can be assumed that the robot wheels roll without slipping and no sinkage occurs. These conditions are commonly modeled with holonomic and nonholonomic kinematic constraints (e.g., [29]). However, such assumptions are often violated when the vehicle moves on soft terrain. In this case, mobility can be understood in the sense of the ability to move away from a certain configuration or to move with maximum speed. This definition is close to the trafficability concept introduced by Apostolopoulos [2], which points to the capacity of the vehicle to overcome terrain resistance and generate traction.

There is also a lack of consensus regarding which strategies are the best to enhance the mobility of wheeled robots operating on unstructured terrain. Reduction of the slip at the wheelterrain contact area has been proposed in several works as a means to achieve this objective [24, 34]. In these papers, the interaction of wheel with hard ground is modeled using the assumption of Coulomb friction while the ratio of tangential to normal forces at the wheel-ground contact is minimized with the goal of reducing the risk of developing slip. While not directly dealing with soft soil modeling, these papers highlight the need for keeping wheel slip under control in order to improve the vehicle behavior. When soft terrain enters into the picture, the phenomena at the wheel-terrain interface become more complex and Coulomb friction models can no longer be used to describe them accurately. Then, two options are left to predict the effect of design and actuation parameters on robot mobility. The first one is turning to detailed models of the contact interface. These models are typically used in forward-dynamics simulation settings and require an accurate knowledge of the set of parameters that characterize the terrain properties, which are not always accessible. The second option consists in finding design and operation guidelines of general validity. These can offer simple means to compare alternative designs and can be used to define objective functions for design, operation, and control.

Based on results obtained from experiments with a four-wheeled rover, Ishigami stated that 
a variation in the normal force distribution does not change the drawbar pull developed by the vehicle [21]. However, it is acknowledged in the same work that a balanced load distribution helps reduce the resistant torque on some wheels and consequently improve the rover mobility. On the other hand, some researchers mention a uniform distribution of normal forces among the factors that enhance mobility. Grand et al. state that balancing the normal loads helps the vehicle to develop higher drawbar pull [18]. Along the same lines, Freitas et al. suggested that uniformly distributing the weight of the rover among the wheels is a valid strategy to achieve better mobility, when adequate information about contact forces is not available [10]. A similar conclusion was reported by Michaud et al. [28]: the load distribution among the wheels has to be even on flat ground to achieve the best performance. Kuroda et al. [23] and Kubota and Naiki [22] also reported that a relation exists between the performance of rovers on rigid ground and their normal force distribution. As a consequence, special attention must be paid to good adaptation to the terrain and the position of the center of mass (CoM) when deciding on the rover structure and configuration. The normal load and the motor torque applied to each wheel were computed by Iagnemma and Dubowsky [20] as the solution of an optimization problem to enhance mobility for quasi-static motion of the rover on rough terrain. In the work by Thueer and Siegwart the likelihood of incurring wheel slip was reduced by minimizing the virtual friction coefficient $\mu^{*}=F_{t} / F_{n}$ for all the wheels, where $F_{t}$ is the traction and $F_{n}$ the normal force at the wheel-ground interface [35]. Following this approach on homogeneous soft terrain, traction is maximized if the normal forces are the same for all the wheels. An experimental confirmation of these statements for a particular rover design was reported by the authors in [13] and [17].

The level of slip must be considered together with the terrain reaction forces when studying mobility. Reaching higher slip values can be used as strategy to develop more traction, e.g., by applying a greater driving torque to the wheels. However, the total drawbar pull goes down when the slip ratio exceeds a certain value [38]. In the tests reported by Lindemann and Voorhees [25], the engineering models of Spirit and Opportunity were placed on a variableslope-angle platform to measure the climbing ability for different slip ratios. It was found that the drawbar pull-slip curve is highly nonlinear. The actual shape of this plot depends on the nature of the terrain and the actions exerted on it by the wheels.

Some strategies exist to determine the climbing ability of a rover via estimation of the soil parameters and the slip ratio of the wheels [30]. The effect of chassis and wheel design on the climbing ability of rovers was investigated by several researchers such as Ding et al. [9] and Ani 
et al. [1]. However, despite being mentioned several times in the literature as a factor to consider during mobility evaluation, normal force distribution has only been studied in a systematic way by a scarcity of works, e.g., [14] with regard to its role in traction on soft soil. The purpose of the work reported here is to study the relation between the internal force distribution in a robot chassis, more specifically the normal force distribution among the wheels, and the robot mobility. This work is an extension of the one presented in [16] and describes the results in more detail, exploring the validity of the proposed approach with a broader scope. The relation between the normal force at a wheel and the drawbar pull that it can develop was used as a starting point for this study. Besides providing a justification for the effect of normal force distribution on mobility, the operation conditions under which this effect is most critical have been identified in this paper. A general framework, not limited to the study of a specific vehicle design or type of terrain, was adopted to ensure the general validity of the results. The structure of the paper follows: Section 2 describes the interaction between a single wheel and soft terrain, showing the relation between normal and tangential forces developed at the wheel-soil contact. Section 3 introduces a new indicator called Normal Force Dispersion and illustrates its effect on the performance of a six-wheeled rover via simulation studies. Section 4 describes the experimental test setup used in our study and the modifications that we introduced on the rover chassis in order to gain full control on the distribution of normal forces among its wheels. Section 5 provides simulation results to show that Normal Force Dispersion has a considerable effect on improving rover performance. Section 6 reports the results of a set of drawbar-pull tests on the rover prototype that confirm the effectiveness of the performance indicator defined in this work. Section 7 introduces the parameters that have the most relevant effect on the relation between the rover mobility and the load distribution among its wheels. Section 8 extends the study to more general scenarios and provides a methodology to achieve better mobility in those cases. Finally, the summary and conclusions of the work are provided in Section 9.

\section{Analysis of single-wheel motion on soft soil}

The interaction between a rigid wheel and soft soil under steady-state conditions is commonly modeled using terramechanics relations [36]. Following this approach, the forces at the wheelterrain interface, shown in Fig. 1, can be obtained as functions of the vehicle state (generalized coordinates and velocities) and a set of parameters that defines the terrain physical properties. 


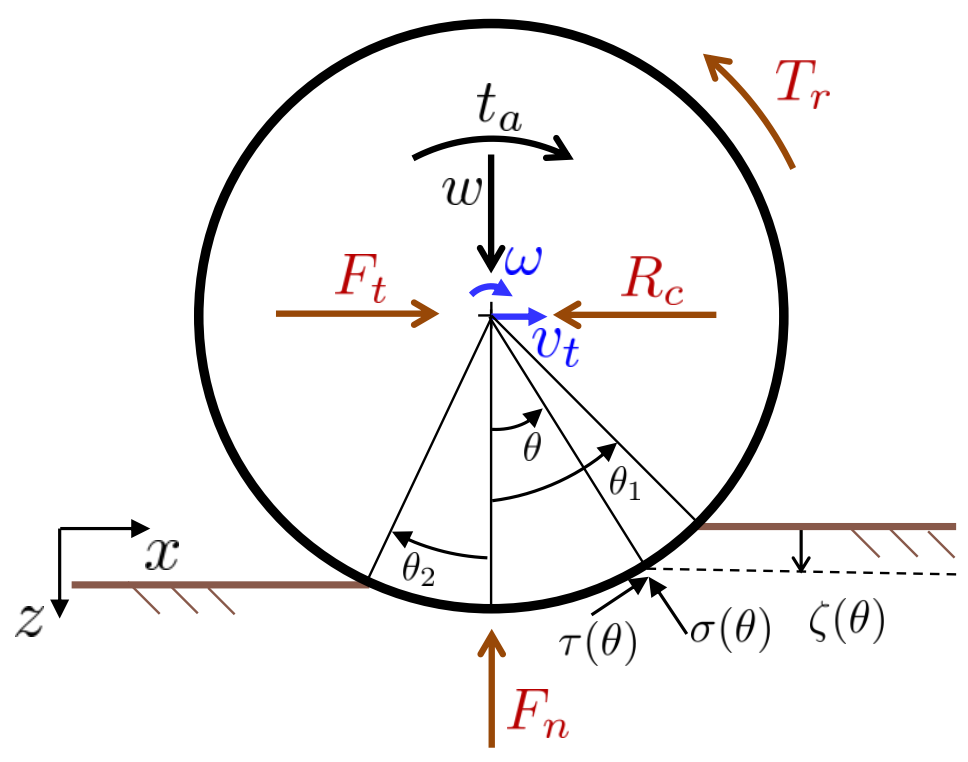

Figure 1: Terrain reactions on a driven single wheel on soft soil, with driving torque $t_{a}$ and normal load $w$

The normal stress at the wheel-terrain interface can be modeled as:

$$
\sigma(\theta)=\left(\frac{k_{c}}{b}+k_{\phi}\right) \zeta(\theta)^{n}
$$

where angle $\theta$ corresponds to the location of any point in the wheel-soil contact patch, $b$ is the wheel width, $n$ is the sinkage exponent, $\zeta$ is the vertical sinkage at any point on the contact surface, and $k_{c}$ and $k_{\phi}$ denote the pressure-sinkage moduli associated with the soil cohesive and frictional components, respectively. For operation on inclined surfaces, the sinkage and the related velocity component must be measured in the direction normal to the ground [21]. The terrain reactions include traction force $F_{t}$, rolling resistance $R_{c}$, normal force $F_{n}$, and resisting moment $T_{r}$.

The shear stress can be determined as

$$
\tau(\theta)=[c+\sigma(\theta) \tan \phi]\left[1-e^{-r\left[\theta_{1}-\theta-(1-s)\left(\sin \theta_{1}-\sin \theta\right)\right] / K}\right]
$$

where $c$ is the terrain cohesion, $\phi$ the internal friction angle, $r$ the wheel radius, $K$ the shear deformation modulus, and $s$ the wheel slip ratio, defined as $s=\left(r \omega-v_{t}\right) / r \omega$, where $\omega$ is the angular velocity of the wheel and $v_{t}$ the velocity of its center.

If the above quantities are known, then the normal force at each wheel and the drawbar pull 
(DBP), $F_{D}=F_{t}-R_{c}$, that the wheel is able to develop can be derived from the terramechanics expressions, namely

$$
\begin{aligned}
& F_{D}=r b\left(\int_{\theta_{2}}^{\theta_{1}} \tau(\theta) \cos \theta d \theta-\int_{\theta_{2}}^{\theta_{1}} \sigma(\theta) \sin \theta d \theta\right) \\
& F_{n}=r b\left(\int_{\theta_{2}}^{\theta_{1}} \tau(\theta) \sin \theta d \theta+\int_{\theta_{2}}^{\theta_{1}} \sigma(\theta) \cos \theta d \theta\right)+c_{z} \dot{\zeta}
\end{aligned}
$$

where $\theta_{1}$ is the entry angle and $\theta_{2}$ the exit angle as shown in Fig. $1, c_{z} \dot{\zeta}$ is a modification from the original form, introduced by Azimi et al. [4], $c_{z}$ is a damping coefficient, and $\dot{\zeta}$ is the sinkage velocity.

The relation between $F_{D}$ and $F_{n}$ can be obtained from Eqs. (3) and (4). Through this relation it is possible to establish how a change in the normal force affects the mobility. Equation (2) shows that the shear stress $\tau$ increases with the normal stress $\sigma$. The relation between the two is nonlinear, as it includes an exponential term that is, in turn, a function of the slip $s$. This implies that the relation between $F_{D}$ and $F_{n}$ is nonlinear as well. Wheel slip and soil properties provide the necessary parameters to define this relation.

\subsection{DBP and normal force relation}

The effect of slip and soil parameters on the relation between drawbar pull and normal force was studied using the Generic Multibody Dynamics Library (GMDL), an in-house developed multibody software tool [12].

A set of simulation tests of the motion of a single wheel moving on soft terrain was carried out using terramechanics relations to model the wheel-terrain interaction. The wheel properties, mass $m=2.55 \mathrm{~kg}$, radius $r=0.175 \mathrm{~m}$, and width $b=0.15 \mathrm{~m}$, were chosen to match those of the rover prototype described in Section 3.1. The slip at the wheel-ground interface was specified by kinematically guiding the wheel upon setting the velocity of the wheel center $v_{t}$ and its angular velocity $\omega$ in planar motion. The total load $F_{n}$ supported by the wheel was adjusted for each simulation test, within a range of up to $600 \mathrm{~N}$. The simulation output was the drawbar pull developed at each numerical test.

Table 1 shows two sets of soil parameters used for the terramechanics relations. Type "A" is 


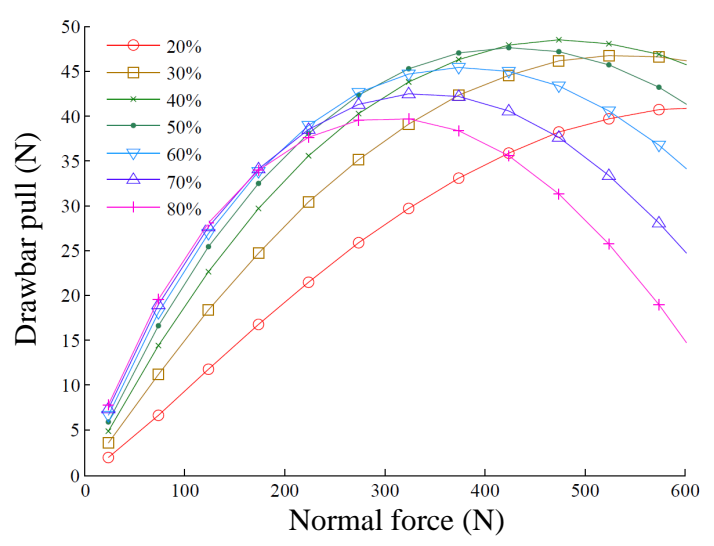

(a) $k_{\phi}=820 \mathrm{kN} / \mathrm{m}^{n+2}$

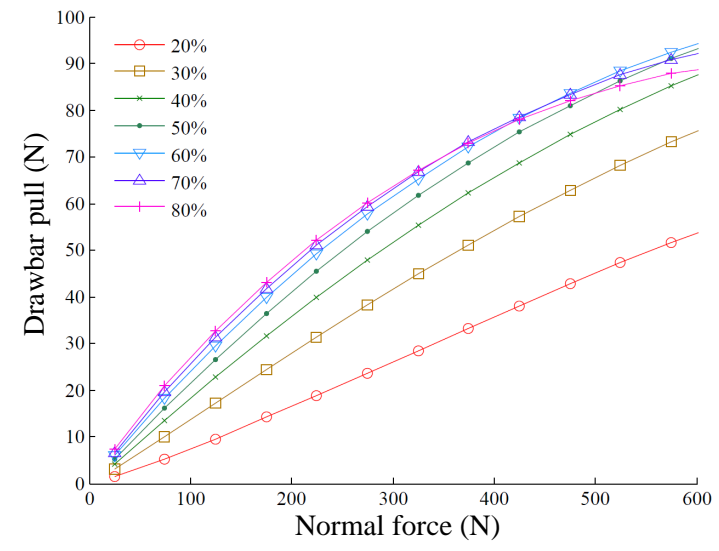

(b) $k_{\phi}=2000 \mathrm{kN} / \mathrm{m}^{n+2}$

Figure 2: The $F_{D}$-vs.- $F_{n}$ curves for a single wheel with different slip ratios

Table 1: Set of soil parameters used for the study of $F_{D}$-vs.- $F_{n}$ curves

\begin{tabular}{lllllll}
\hline Soil type & $n$ & $c$ & $\phi$ & $k_{c}$ & $k_{\phi}$ & $K$ \\
\cline { 2 - 6 } & $(-)$ & $\left(\mathrm{N} / \mathrm{m}^{2}\right)$ & $(\mathrm{deg})$ & $\left(\mathrm{N} / \mathrm{m}^{n+1}\right)$ & $\left(\mathrm{kN} / \mathrm{m}^{n+2}\right)$ & $(\mathrm{m})$ \\
\hline A & 1 & 220 & 33.1 & 1400 & 2000 & 0.015 \\
B & 1 & 220 & 33.1 & 1400 & 820 & 0.015 \\
\hline
\end{tabular}

an estimation of the properties of the terrain used in the experiments with the Rover Chassis Prototype (RCP), which are described in detail in Section 4. Soil "A" parameters were identified using an extensive set of drawbar-pull experiments. The data yielded by these allowed to relate the drawbar pull developed by the rover to the slip ratio of the wheels. Each experiment was simulated later in a forward-dynamics setting, using terramechanics relations to model the wheel-terrain interaction. The terrain parameters were then identified by curve-fitting, comparing experimental and simulation results. More details on the process of soil parameter identification required for the RCP simulation are given in [14] and [11]. Type "B" is a theoretical variation of type "A", from which it only differs in the value of $k_{\phi}$. Although soil "B" may not exist in reality, it is still useful to define it to study the effect of $k_{\phi}$ on system behavior via simulation.

The $F_{D}$-vs.- $F_{n}$ curves for soils "A" and "B" are shown in Fig. 2 . These plots show that for low values of $F_{n}$ the relation between normal force and drawbar pull is practically linear for the entire range of slip ratios. On the other hand, higher normal loads on the wheel and higher slip, e.g., over 50\%, bring along significant deviations from linearity. These are accentuated by low values of the $k_{\phi}$ modulus, which was found by simulation studies to be the most influential of 
all terrain parameters with regard to the curvature of the plotted lines [14].

\section{Normal force dispersion as mobility indicator}

The mobility of a wheeled robot depends on its ability to generate a required amount of drawbar pull while keeping the slip ratio low. The discussion in the previous section points out that the normal force at each wheel of a robot affects the tangential force developed and, in turn, the total drawbar pull that the vehicle provides. The terrain normal reactions have to balance the inertial and external forces applied on the rover. However, changing the normal load distribution among the wheels can result in different values of the total drawbar pull developed by the vehicle. The effect of normal force distribution can be studied using the $F_{D}$-vs.- $F_{n}$ curve. An example for a planar three-axle system in $2 \mathrm{D}$ motion is shown in Fig. 3 , where the $F_{D}$-vs.- $F_{n}$ curve has a generic nonlinear shape similar to those in Figs. 2(a) and 2(b).

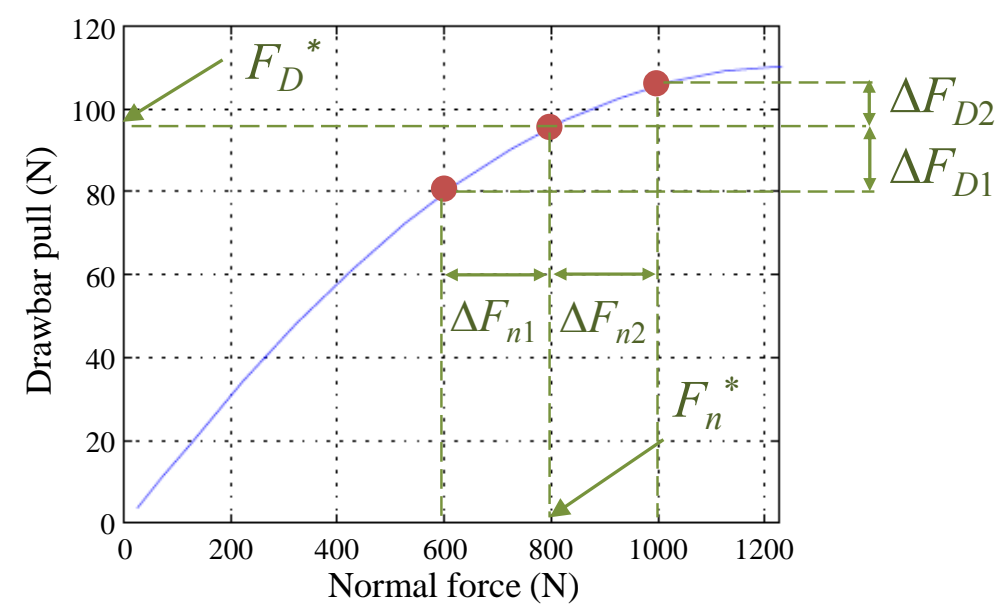

Figure 3: Effect of non-uniform normal force distribution on the total available drawbar pull

If the three axles are moving with the same angular speed, and the terrain under the vehicle is homogeneous, then the same curve can be used for all the wheels. In this case, an even normal load distribution would be the one in which $F_{n 1}=F_{n 2}=F_{n 3}=F_{n}^{*}$. A normal load transfer between the first and second axles of the robot $\left(\Delta F_{n 1}=-\Delta F_{n 2}\right)$ will result in $\Delta F_{D 1}<0$ and $\Delta F_{D 2}>0$ in the drawbar pull at these wheels. If the slope of the $F_{D}$-vs.- $F_{n}$ curve decreases consistently with $F_{n}$, i.e., the curve is sublinear, then $\left|\Delta F_{D 2}\right|<\left|\Delta F_{D 1}\right|$, which will yield a lower total drawbar pull available for the same slip values. In other words, in the uneven configuration the slip should become higher in order to achieve the same drawbar pull delivered by its 
balanced counterpart, where the normal forces are uniformly distributed among the wheels.

For the case of a wheeled robot operating on homogeneous terrain, the $F_{D}$-vs.- $F_{n}$ relation will be the same for all the wheels if they are identical and have the same slip. These assumptions can be considered close enough to reality for a broad range of operating conditions.

We define here a performance indicator termed the Normal Force Dispersion (NFD), denoted by $\eta$, to measure and quantify the uniformity of the normal force distribution. This performance indicator is the standard deviation of the normal forces at the wheel-terrain contact interfaces, namely,

$$
\eta\left(F_{n 1}, \ldots F_{n p}\right)=\sqrt{\frac{1}{p} \sum_{i=1}^{p}\left(F_{n i}-\mu\right)^{2}}
$$

where $p$ is the number of wheels of the vehicle and $\mu$ is the average normal force:

$$
\mu=\frac{1}{p} \sum_{i=1}^{p} F_{n i}
$$

An even distribution of normal forces $\left(F_{n 1}=F_{n 2}=\ldots=F_{n p}\right)$ would result in $\eta=0$, which is optimum in terms of developed drawbar pull for operation on homogeneous terrain and assuming that all the wheels of the vehicle have the same slip ratio. Quantifying the unevenness of the load distribution via NFD facilitates the comparison of different rover configurations in terms of their mobility, while it may avoid the need for a detailed knowledge of the terrain properties. The exact value of the terrain reaction is not required since only information on the relative change in the performance of the rover is enough to make design and operation decisions. The index can be used to determine which configuration produces the most uniform load distribution among the wheels for a given maneuver or operation.

As a conclusion, it can be stated that making the normal force distribution more uniform will have a noticeable effect on the drawbar pull when the $F_{D}$-vs. $-F_{n}$ curve shows an apparent sublinear relationship. This is the case of operation conditions where high slip values are expected to develop, such as in slope climbing, or in the presence of loose terrain with low values of $k_{\phi}$. In other situations, with an almost linear $F_{D}$-vs.- $F_{n}$ plot, such as when moving on flat ground or climbing mild slopes on cohesive terrain, the uniformity of the normal load distribution will still have a positive effect on rover mobility, but this may not be very significant. 


\subsection{Application of the concept}

The NFD was used to study the mobility of the RCP, a six-wheeled rover prototype developed by the Robotics and Automation unit of MDA (MacDonald, Dettwiler and Associates Ltd.) shown in Fig. 4(a). The rover was built as an engineering model for various experimental purposes.

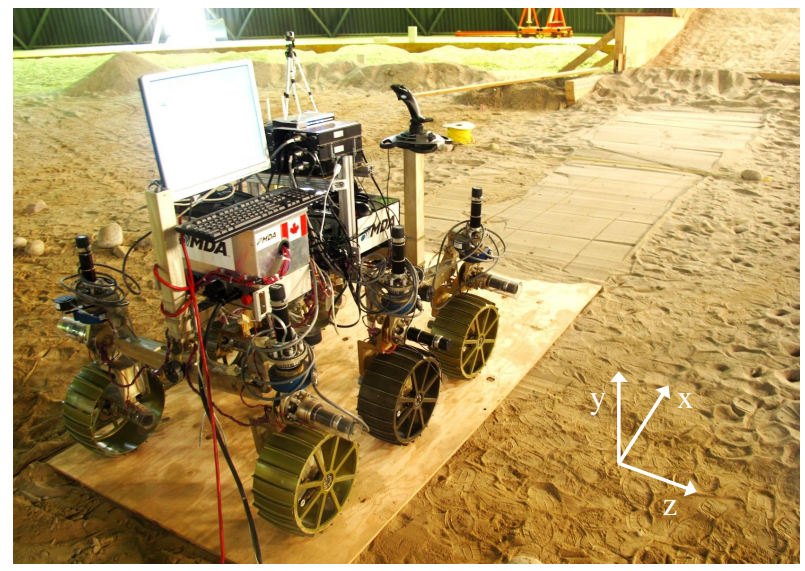

(a) Actual rover prototype used in the experiments

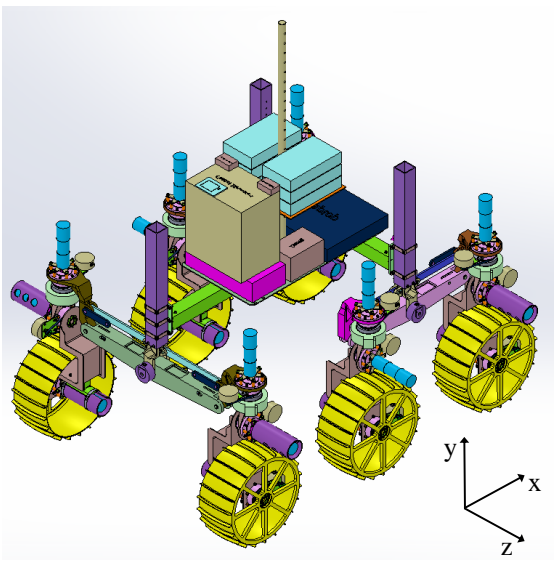

(b) CAD model

Figure 4: The Rover Chassis Prototype

The rover main body is attached to three bogies—starboard, port, and rear-via passive revolute joints, which enables free rotation of the bogies with respect to the body. Each bogie is connected to two legs, and each leg is connected to a wheel. The wheel joints are actuated by DC motors controlled with angular velocity commands. The rover is approximately $1.4 \mathrm{~m}$ long and $1 \mathrm{~m}$ wide, with a total mass of nearly $125 \mathrm{~kg}$. The wheels are metallic cylinders of radius $r=0.175 \mathrm{~m}$, with grousers.

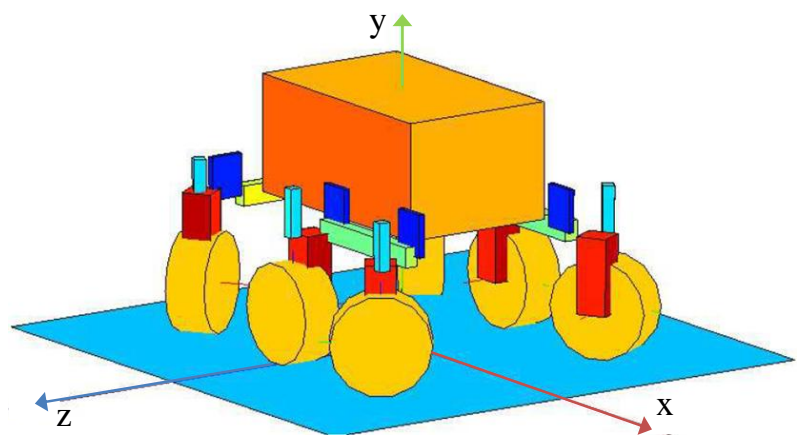

Figure 5: Multibody model of the RCP built with the GMDL

A full-scale model of the rover, shown in Fig. 5, was developed using the GMDL. The multibody model of the rover is composed of 28 rigid bodies connected by 27 revolute joints. The total number of constraint equations is 183 . The multibody library includes functions to evaluate the 
wheel-terrain interaction forces according to the terramechanics semi-empirical relations introduced in Eqs. (3) and (4). For the simulation tests performed in this study, an index-1 augmented Lagrangian formulation with position and velocity projections [5] was employed together with the Newmark integration formulas [31].

\subsection{Effect of normal force distribution on wheel slip}

The NFD defined in Eq. (5) is to be used as a performance indicator of the mobility of a wheeled robot. According to the previous discussion in this section, of two different configurations of the same vehicle, the one with a more uniform normal load distribution will develop a lower slip to provide the same drawbar pull. The simulation results in this section support this statement.

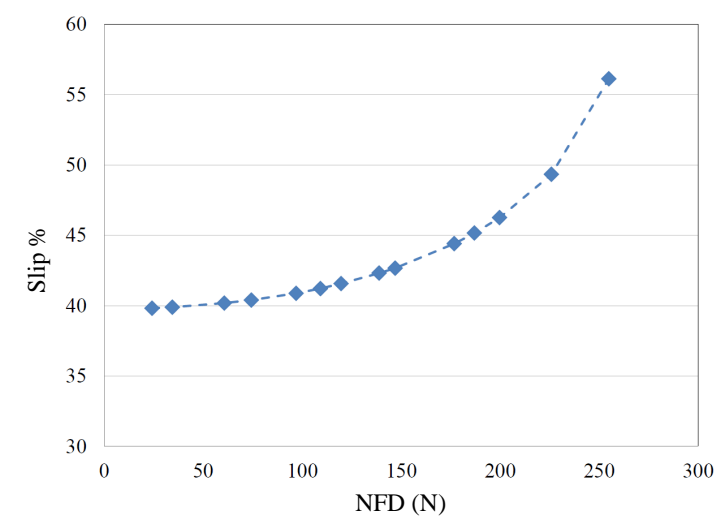

(a) Slip developed when climbing a $10^{\circ}$ slope, with a 22.5-kg payload

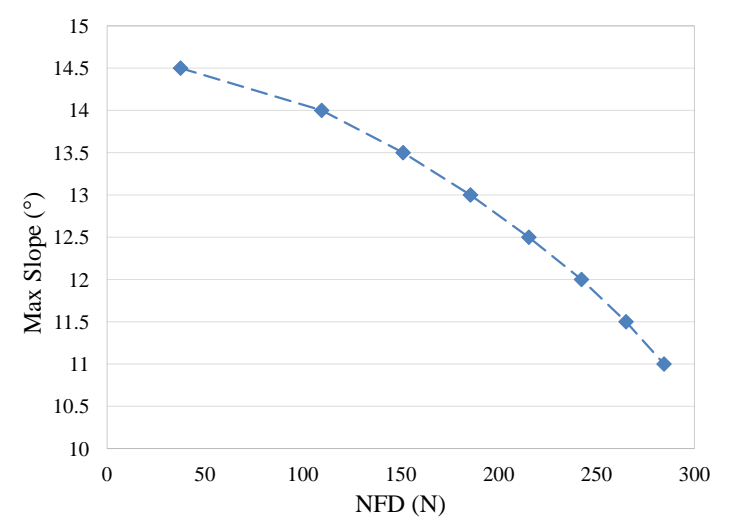

(b) Maximum slope angle that the RCP can climb with $90 \%$ slip

Figure 6: Performance of the RCP predicted by the simulation with the GMDL

First, the climbing maneuver of the RCP on a $10^{\circ}$ slope with the properties of soil type "A" from Table 1 was simulated. The wheels of the rover were commanded to move with a constant angular speed $\omega=0.4 \mathrm{rad} / \mathrm{s}$. In order to obtain different load distributions among the wheels of the RCP, a movable 22.5-kg payload was added to the rover model. The simulation was repeated for different locations of the payload along the longitudinal axis of the vehicle. This resulted in variations of the position of the CoM of the rover, which in turn produced different values of NFD during the climbing maneuver.

In Fig. 6(a) the slip developed to climb a $10^{\circ}$ slope while carrying the $22.5-\mathrm{kg}$ payload is plotted for different values of NFD. The results confirm that lower values of NFD resulted in a lower slip required to carry out the climbing, which is beneficial from the mobility and energy- 
consumption points of view.

\subsection{Effect of normal force distribution on drawbar pull}

Alternatively, the improvement in mobility can be quantified by the value of the maximum slope that the vehicle can negotiate. The climbing maneuver was simulated for a variable slope with the properties of soil "A" from Table 1 . The rover was considered unable to climb if the required slip ratio became higher than $90 \%$. A similar slip threshold was used in slope-climbing tests with the Dynamic Test Model of the Mars Exploration Rover [25]. The slope angle was increased until the rover was unable to complete the maneuver without exceeding the maximum admissible slip. Figure 6(b) shows that a correlation exists between the value of NFD and the maximum slope the vehicle can successfully climb.

Heavy normal loads and low values of the $k_{\phi}$ coefficient of the terrain were identified in Section 2.1 as the most influential factors on the $F_{D}$-vs. $-F_{n}$ relation. Their effect on the slip developed by the RCP during a climbing maneuver is described in Section 7.

\section{The RCP and test setup}

Results in Section 3 showed the effect of repositioning the CoM on the normal force distribution, which in turn influences rover mobility. The rover ability to develop drawbar pull can be evaluated by slope-climbing maneuvers, as explained above. Drawbar-pull tests can be considered analogous to slope-negotiation tests if the line of action of the applied force is close to the rover CoM, at least in the vertical direction. The action of gravity along the main longitudinal axis of the rover during slope climbing has effects similar to an external drawbar-pull load applied on the chassis. That way, the ability of the rover to develop drawbar pull on flat terrain can be used as an indication of its ability to climb slopes. Drawbar-pull experiments are also easier to carry out, as they require less resources than building a variable-angle, soft-soil slope.

A set of experiments, including drawbar-pull tests with variable NFD and wheel slip, was carried out with the RCP on soft, sandy soil at UTIAS (University of Toronto Institute for Aerospace Studies) Mars Dome. Large areas covered by sand made this facility suitable for testing the RCP 
mobility on soft soil. The objective of these experiments was to measure the drawbar pull developed by the rover for a certain wheel slip and load configuration. The slip ratio was kept constant in each experiment by controlling the forward velocity of the rover with a winch, schematically shown in Fig. 7. The wheels of the vehicle were commanded to move with a constant angular velocity of $0.4 \mathrm{rad} / \mathrm{s}$.

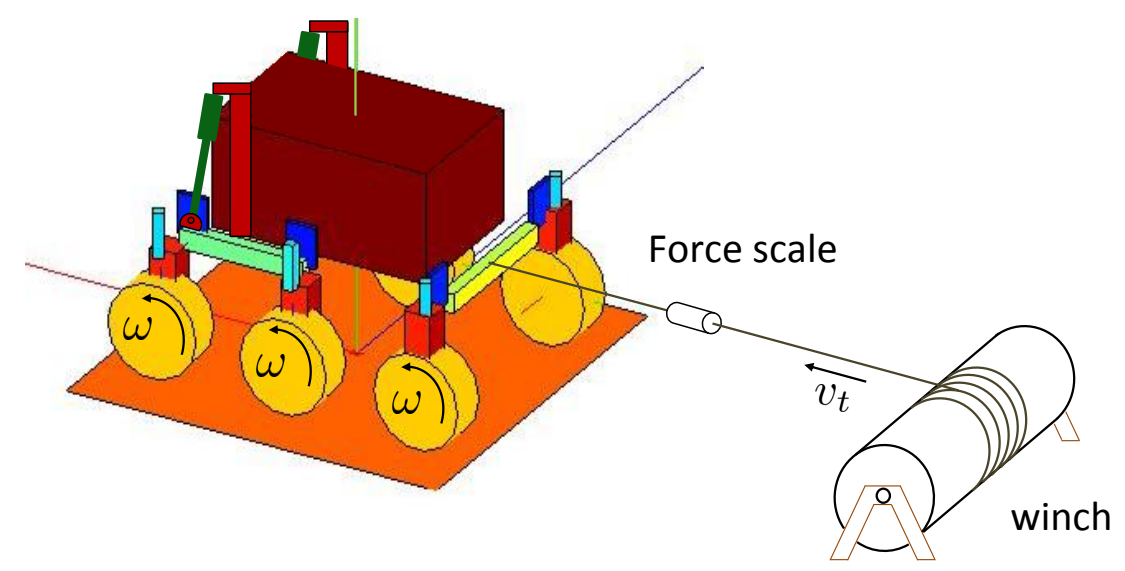

Figure 7: Winch used to control the translational velocity of the rover

The RCP is equipped with six force-torque sensors mounted on each of its legs. The normal, tangent, and lateral terrain reactions acting on each wheel were measured by these triaxial sensors during each experiment. Additionally, the tension on the winch rope was recorded to provide redundant measurements, as shown in Fig. 8.

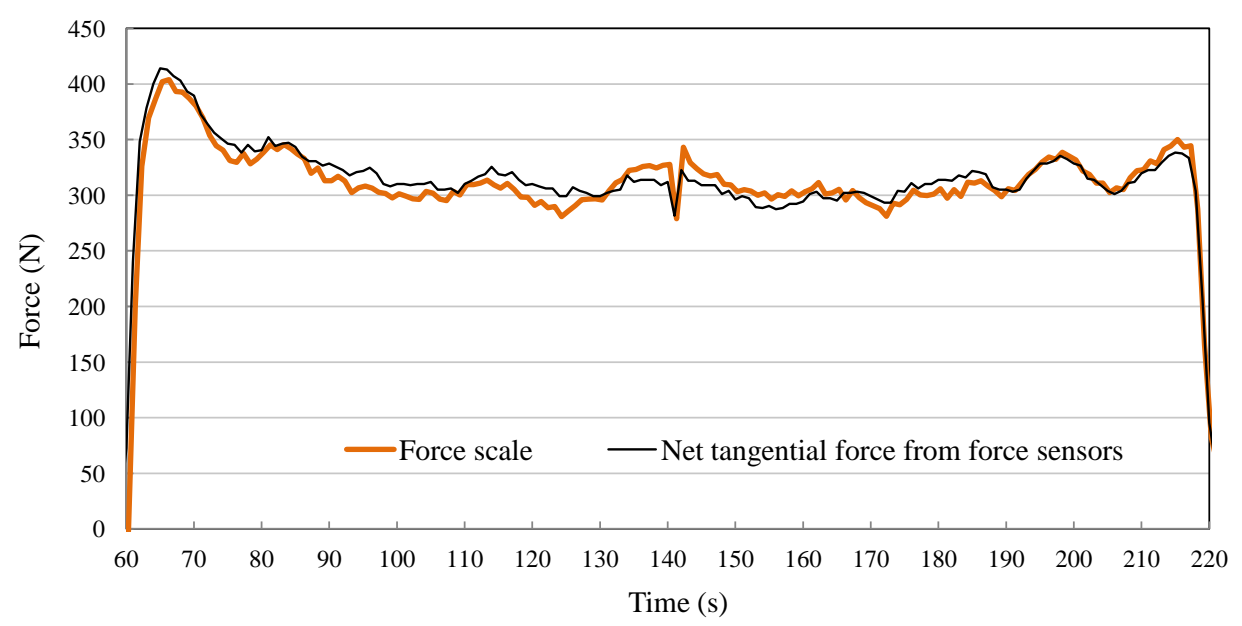

Figure 8: Comparison of the force applied by the winch and the net tangent force measured by the on-board force sensors 


\subsection{Modification of load distribution among the wheels}

The NFD can be reduced significantly via design considerations, e.g., with a suitable distribution of the batteries and other heavy elements on the platform. Load distribution can also change during operation, e.g., by repositioning movable components of the rover such as manipulators.

To represent this with the RCP, and in order to obtain different sets of normal force distributions for each experiment, two movable $11.25-\mathrm{kg}$ mass elements were mounted on the rover platform. Two attachment positions for the mass elements were designated on the rover body: the front attachment was the front tip of the main body and the rear attachment was the connection between the rear bogie and the main body. Three different configurations were achieved by distributing the mass elements between these two attachments. The mass-at-front configuration (Front_Config) corresponds to placing both mass elements at the front attachment. The mass-atrear configuration (Rear_Config) refers to the one in which both mass elements were located at the rear attachment. The mass-in-the-middle configuration (Middle_Config) was obtained when one mass element was located at the front attachment and the other at the rear attachment.

In practice, a uniform load distribution among the wheels of the RCP cannot be achieved only via the relocation of the CoM due to limitations in the weight and the location of the extra mass elements. Furthermore, due to the presence of passive joints in the RCP suspension, full control of the load distribution among the wheels is not possible. This is a feature commonly found in planetary exploration rovers. By repositioning the CoM of the rover only the load distribution between the rear wheels and the side bogies can be controlled. The load distribution between the front and middle wheels on the side bogies depends on the orientation of the bogie with respect to the rover body, which cannot be controlled because the body-bogie joint is passive. We will use the term redundant internal actuation to refer to the actuation introduced in these originally passive joints of the rover suspension. Besides modifying the normal force distribution among the vehicle wheels, redundant internal actuation can also be used to enhance certain maneuvers like obstacle negotiation. Moreover, the actuation strategy can be modified during operation.

The effect of CoM repositioning and redundant internal actuation is illustrated with two configurations of the RCP in Fig. 9, where Fig. 9(a) represents the default configuration of the RCP. In Fig. 9(b), the CoM position is shifted towards the front of the rover and redundant internal actuation is introduced between each side bogie and the main body. In this example, 


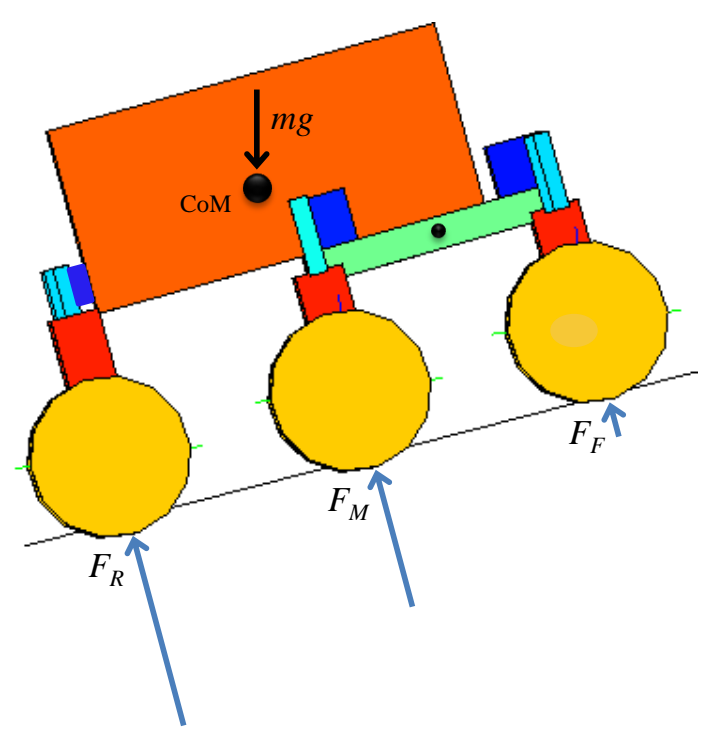

(a) Default configuration

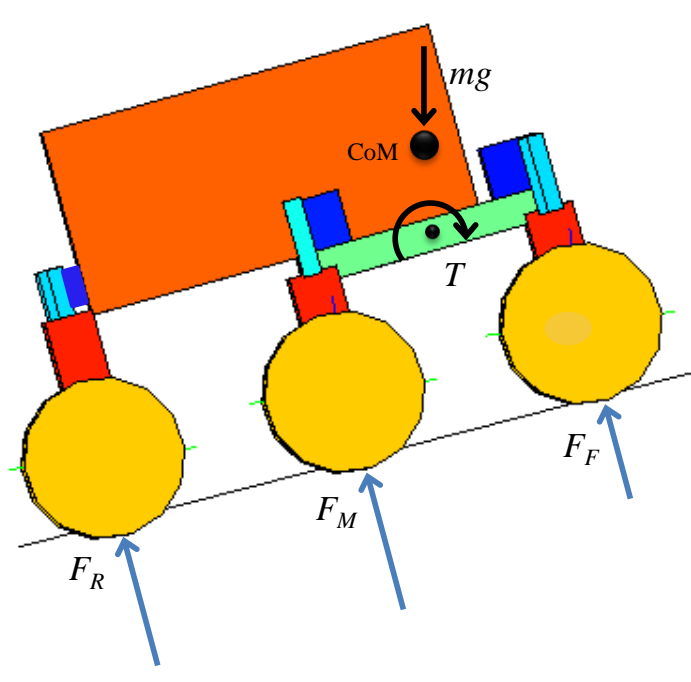

(b) Improved configuration with lower NFD

Figure 9: Effect of CoM repositioning and redundant internal actuation on normal force distribution

a $60 \mathrm{Nm}$ torque in the clockwise direction when viewed from outside of the rover is applied at each bogie joint. In these figures the lengths of the arrows that represent the reactions at the wheel-terrain interface are proportional to the magnitudes of the normal forces obtained from simulation. In the default configuration in Fig. 9(a), the resultant load distribution gives $\eta=158.1 \mathrm{~N}$. The rover in this configuration is able to negotiate a maximum slope of $11^{\circ}$. As shown in Fig. 9(b), a considerable reduction of NFD was achieved with the application of the techniques described here. The NFD went down to $\eta=24.2 \mathrm{~N}$ and the rover was able to climb a $14.5^{\circ}$ slope with the same slip as in the original configuration.

Redundant internal actuation was introduced in the RCP by means of prismatic actuators mounted between the rover chassis and the port and starboard bogies [27], as shown in Fig. 10. The design of these actuators was carried out considering that they should not limit the range of motion of the rover in maneuvers such as obstacle climbing, as shown in Fig. 11; in fact the authors have reported that redundant internal actuation can also assist climbing maneuvers [15]. Actuators were designed to provide a maximum torque of $60 \mathrm{Nm}$ about the bogie articulation. 


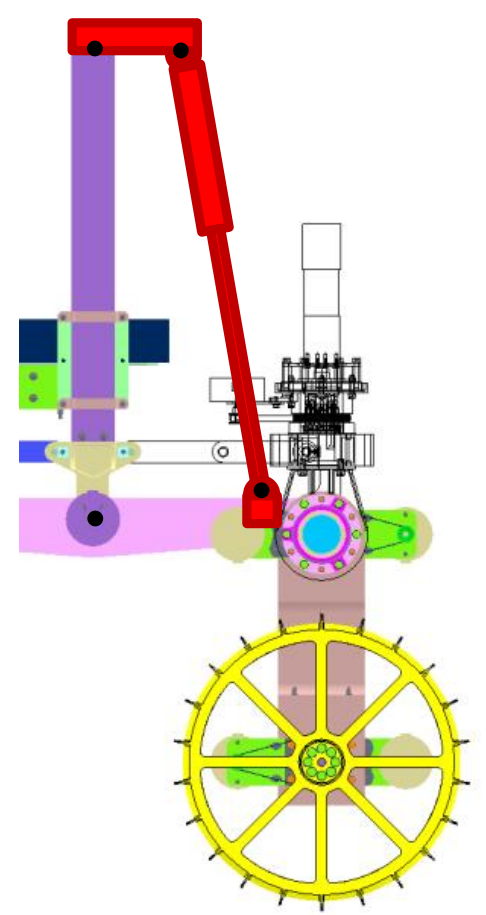

(a) Schematic of the actuation mechanism

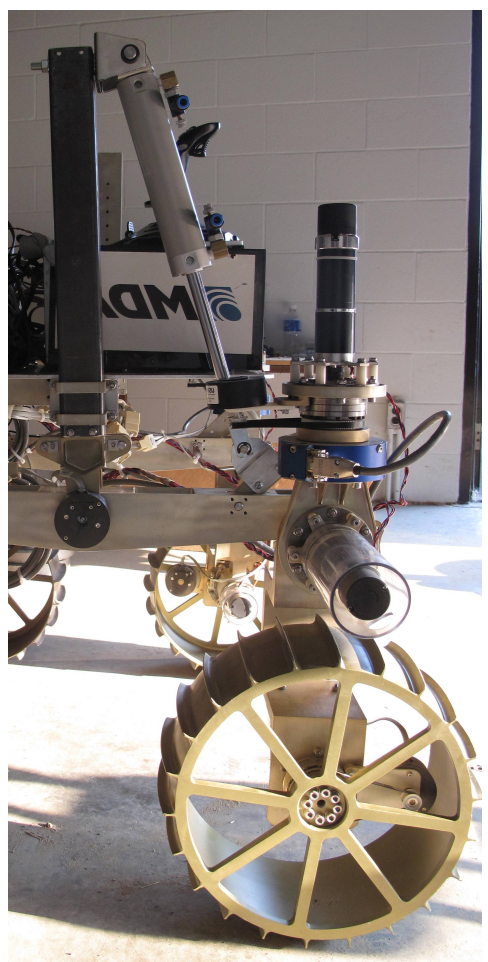

(b) RCP with the attached pneumatic actuator

Figure 10: Design modification of the RCP to add redundant internal actuation

\section{Improvement of rover performance via modification of the NFD}

The improvement of rover mobility was studied in Sections 3.2 and 3.3 from two different points of view:

- Reduction of the slip ratio required to develop a certain drawbar pull. This applies to operations on flat ground, slopes with a constant angle, or when the rover carries a load.

- Improvement of the ability of the rover to develop higher drawbar pull for a certain slip ratio.

The first scenario is more likely to happen as the slip is usually not specified, but rather developed; the slip increases until the wheel can provide the required drawbar pull for the required motion, although the DBP-slip relation is not linear and the drawbar pull can decrease beyond a certain value of the slip ratio. The first point of view is studied in this section using only simulation. The second case is easier to realize experimentally and it allows one to carry out 


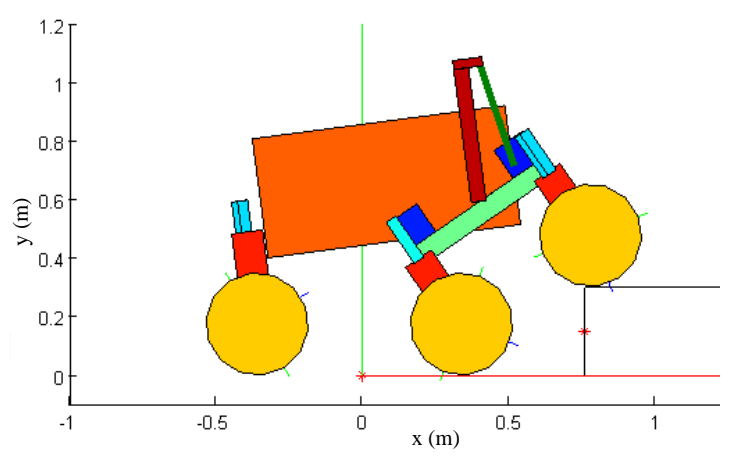

(a) Dynamic simulation

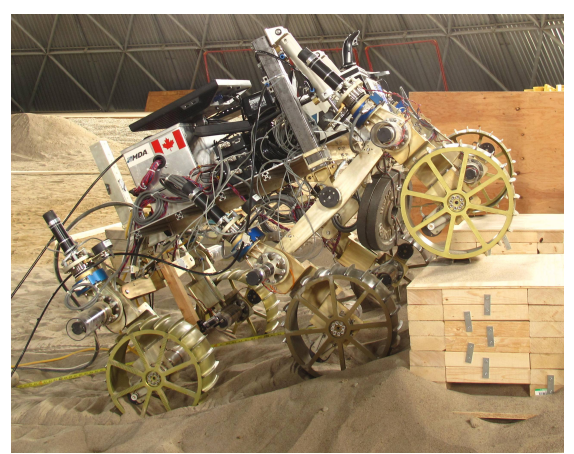

(b) Experiment

Figure 11: Climbing maneuver of the RCP on a 0.3-m-high step with the aid of the actuators

tests involving the continuous measurement of the drawbar pull. This was the approach adopted for the set of experimental tests performed with the RCP. The results of these experiments are reported in Section 6. Here the first case is studied via simulation with the model of the RCP and three slopes, listed in Table 2, which differ only in their frictional coefficients $k_{\phi}$ and $\phi$, and their slope angles. When the rover climbs a slope with high frictional coefficients, the slip developed to complete this maneuver is lower when compared to the slip required to climb slopes with low frictional coefficients. It is desirable to assign a slope angle to each type of terrain so that the slip developed to climb that slope in the absence of redundant internal actuation exceeds $80 \%$. This can facilitate the comparison in the improvement of the rover performance between different cases. Therefore, the slope angle for the soils with higher frictional coefficient was set larger, as shown in Table 2.

Table 2: Properties of different slopes used in the simulation

\begin{tabular}{c|ccccccc}
\hline Case & Slope angle & $k_{\phi}$ & $\phi$ & $n$ & $c$ & $k_{c}$ & $K$ \\
\cline { 2 - 8 } & $(\mathrm{deg})$ & $\left(\mathrm{kN} / \mathrm{m}^{n+2}\right)$ & $(\mathrm{deg})$ & $(-)$ & $\left(\mathrm{N} / \mathrm{m}^{2}\right)$ & $\left(\mathrm{N} / \mathrm{m}^{n+1}\right)$ & $(\mathrm{m})$ \\
\hline 1 & 9 & 820 & 33.1 & 1 & 220 & 1400 & 0.015 \\
2 & 12 & 1410 & 34.1 & 1 & 220 & 1400 & 0.015 \\
3 & 15 & 2000 & 36.1 & 1 & 220 & 1400 & 0.015 \\
\hline
\end{tabular}

It was observed that during climbing, if no redundant internal actuation is applied, the middle wheels support most of the weight of the vehicle. The internal applied torque $T$ on the bogies can then be used to generate a normal load transfer from the middle to the front wheels. The torque can be modified during the rover operation, based on the motion requirements. Details of the simulation of the RCP climbing slope 2 in Table 2 are included below. 


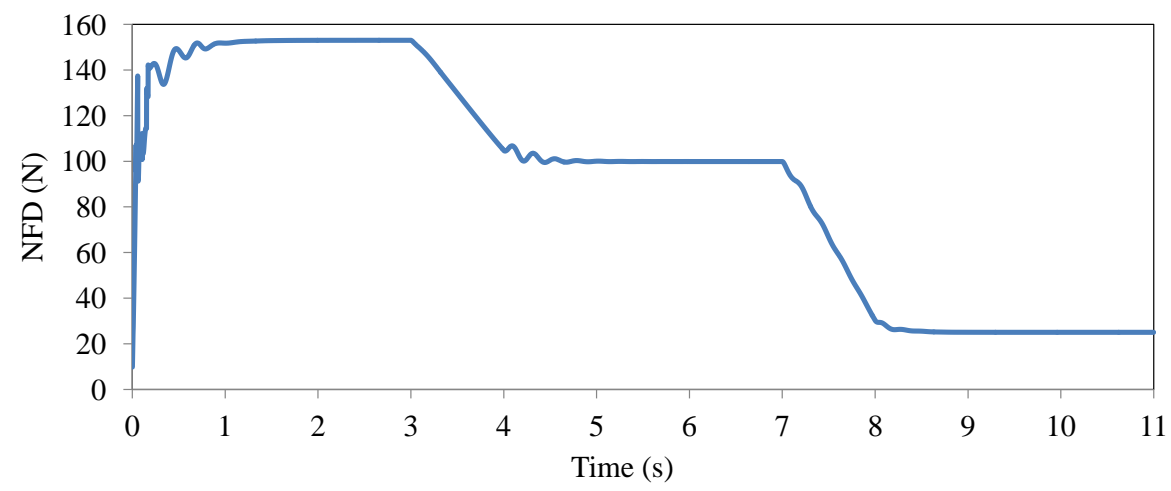

Figure 12: The NFD of the RCP during climbing of terrain 2 in Table 2. The internal actuation torque $T$ was increased in two successive steps, from $0 \mathrm{Nm}$ to $20 \mathrm{Nm}$ and then to $50 \mathrm{Nm}$.

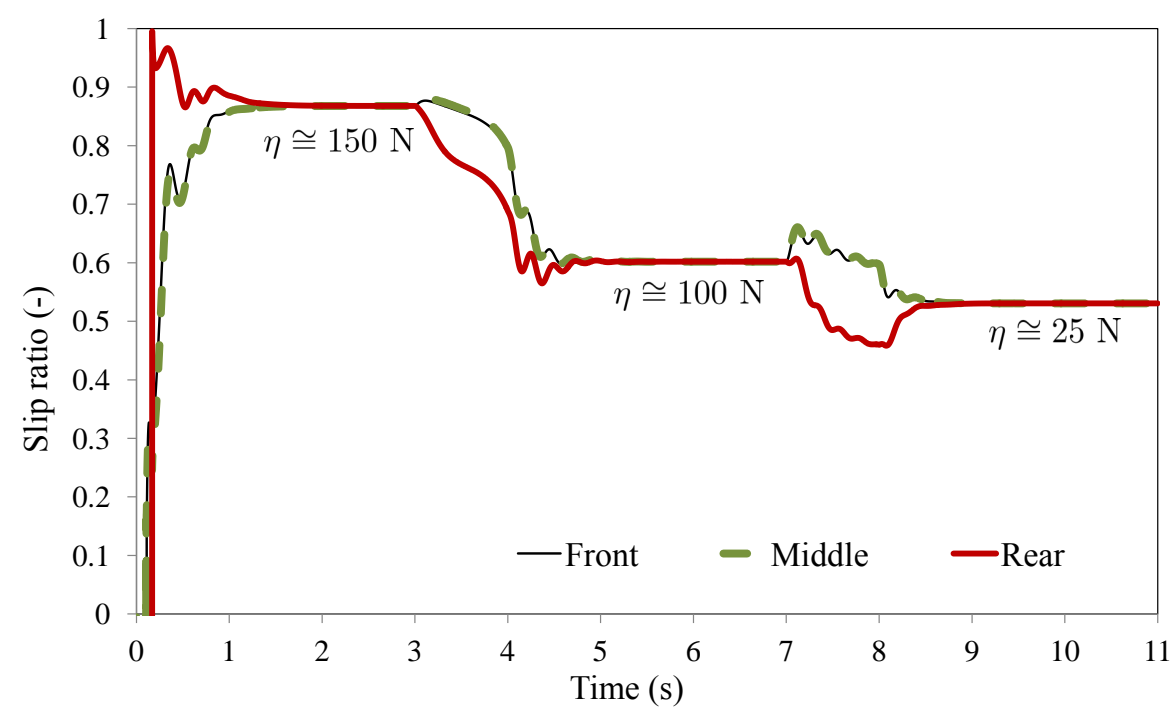

Figure 13: Slip of the RCP when climbing slope 2 in Table 2, for the maneuver in Fig. 12

At the beginning of the simulation the rover was placed on a $12^{\circ}$ slope and the wheels where commanded to move with $\omega=0.4 \mathrm{rad} / \mathrm{s}$. Initially, in the absence of redundant internal actuation, the NFD was $\eta=153 \mathrm{~N}$. The rover reached a steady-state motion after $t=2 \mathrm{~s}$, requiring $87 \%$ slip to move forward. At $t=3 \mathrm{~s}$, the torque on the bogie joint was increased gradually up to $T=20 \mathrm{Nm}$. A new steady-state ensued after $t=5 \mathrm{~s}$. The new normal force distribution, $\eta=100$ $\mathrm{N}$, brought the slip down to $60 \%$. An additional increase in $T$ to $50 \mathrm{Nm}$ further improved the load distribution, enabling the rover to climb the same slope with 53\% slip. These results are displayed in Figs. 12 and 13.

Similar tests were conducted for every case described in Table 2. The effect of two different values of $T$ on the wheel slip and the NFD is summarized in Table 3. The system behavior followed the same pattern shown in Figs. 12 and 13. It must be noted that the beneficial effect on traction of improving NFD was more significant in soils with lower friction coefficient $k_{\phi}$; the 
Table 3: Effect of internal actuation on the slope-climbing ability of the RCP for the cases described in Table 2

\begin{tabular}{c|ccc|ccc|ccc}
\hline & \multicolumn{3}{|c|}{ Part 1 } & \multicolumn{3}{c|}{ Part 2 } & \multicolumn{3}{c}{ Part 3 } \\
& $T$ & $\eta$ & slip & $T$ & $\eta$ & slip & $T$ & $\eta$ & slip \\
Case & $(\mathrm{Nm})$ & $(\mathrm{N})$ & $(\%)$ & $(\mathrm{Nm})$ & $(\mathrm{N})$ & $(\%)$ & $(\mathrm{Nm})$ & $(\mathrm{N})$ & $(\%)$ \\
\hline 1 & 0 & 150 & 89 & 20 & 98 & 53 & 50 & 22.4 & 42 \\
2 & 0 & 153 & 87 & 20 & 100 & 60 & 50 & 25.0 & 53 \\
3 & 0 & 158 & 84 & 20 & 105 & 67 & 50 & 24.4 & 60 \\
\hline
\end{tabular}

relevance of this parameter will be discussed in further detail in Section 7.1.

\section{Experimental results}

The simulation results obtained in the previous section were confirmed with experiments. Improvement of the rover performance is studied here from the point of view of increasing the drawbar pull for a given slip ratio. First, the effect of the aforementioned factors on the normal force distribution of the RCP is reported. Next, the resultant performance improvement due to reduction in NFD is discussed.

\subsection{Effect of reconfiguration on normal forces}

A set of experiments, including drawbar-pull tests with variable load distribution was carried out on the soft, sandy soil of the Mars Dome with the experimental setup described in Section 4 and a fixed slip ratio. The angular velocity of the wheels and its translational velocity were set to $\omega=0.4 \mathrm{rad} / \mathrm{s}$ and $v_{t}=0.027 \mathrm{~m} / \mathrm{s}$, respectively, which resulted in a wheel slip close to $60 \%$. The load distribution was modified via CoM repositioning and redundant internal actuation of the rover chassis.

Figures 14 and 15 show the readings from the force sensors in four different tests. These readings are from the starboard side of the RCP during the motion of the rover on a straight line. Figure 14(a) corresponds to the RCP Rear_Config (defined in Section 4.1), in which the 


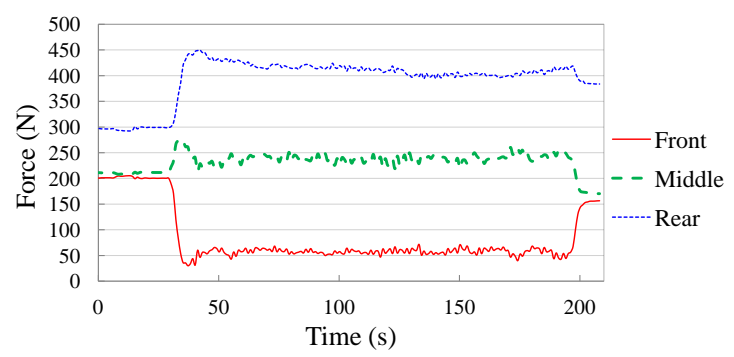

(a) $\eta=139 \mathrm{~N}$

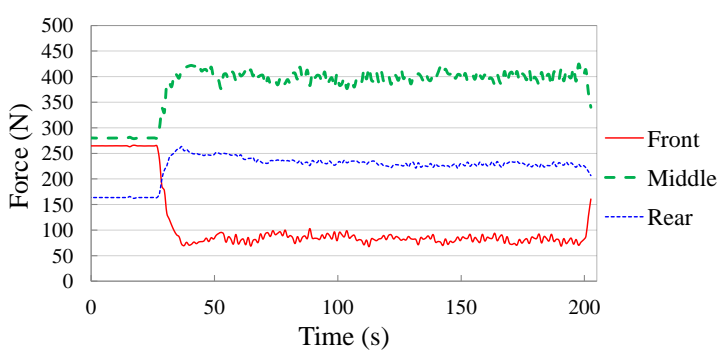

(b) $\eta=113 \mathrm{~N}$

Figure 14: Experimental results: Normal force on the starboard wheels of the RCP with $\eta=139$ $\mathrm{N}$ and $\eta=113 \mathrm{~N}$.

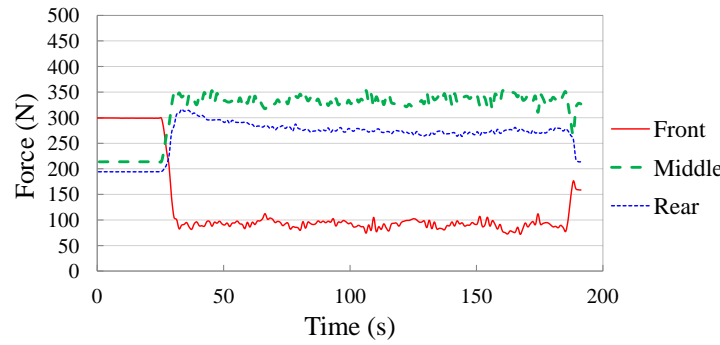

(a) $\eta=97 \mathrm{~N}$

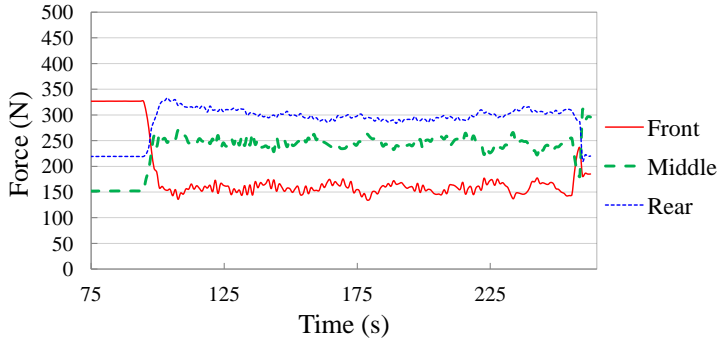

(b) $\eta=69 \mathrm{~N}$

Figure 15: Experimental results: Normal force on the starboard wheels of the RCP with $\eta=97$ $\mathrm{N}$ and $\eta=69 \mathrm{~N}$.

rear wheels support most of the load. The load distribution with this configuration is remarkably uneven with $\eta=139$ N. A second configuration, corresponding to Front_Config was obtained by shifting the mass elements towards the front. The normal forces obtained in this case, which result in $\eta=113 \mathrm{~N}$, are displayed in Fig. 14(b). The comparison of Figs. 14(a) and 14(b) shows that CoM repositioning reduced the load on the rear wheels significantly, transferring it to the side bogies, where the middle wheels supported most of the load.

Next, a $9 \mathrm{Nm}$ torque was applied between the main body and each side bogie. The resulting time history of the normal forces is shown in Fig. 15(a) which is associated with $\eta=97 \mathrm{~N}$. The torque applied on the bogies was relatively small and did not transfer a significant load to the front wheels, but from the middle wheels to the rear wheels. Finally, the redundant internal actuation torque was increased again up to $25 \mathrm{Nm}$ and a higher load from the middle wheels was transferred to the front and the rear wheels, as shown in Fig. 15(b). The NFD was reduced to $\eta=69 \mathrm{~N}$. The comparison of these four experiments confirmed the effectiveness of CoM repositioning and redundant internal actuation in achieving the desired load distribution among the rover wheels. 


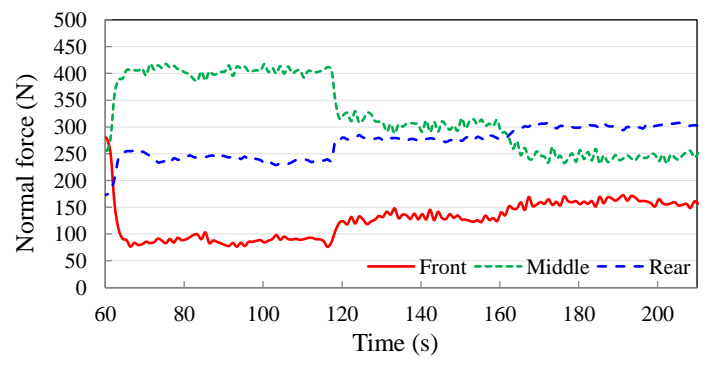

(a) Normal forces obtained in experiment

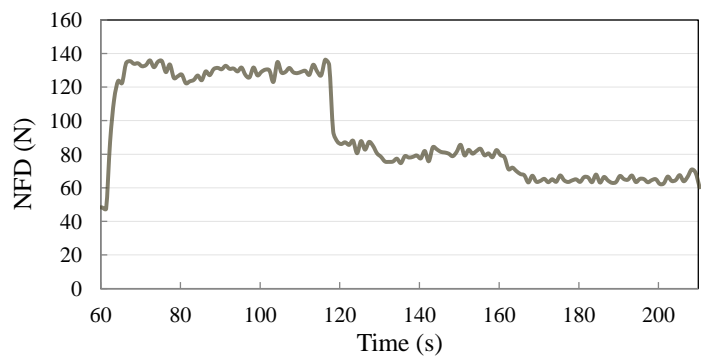

(b) Resultant NFD in experiment

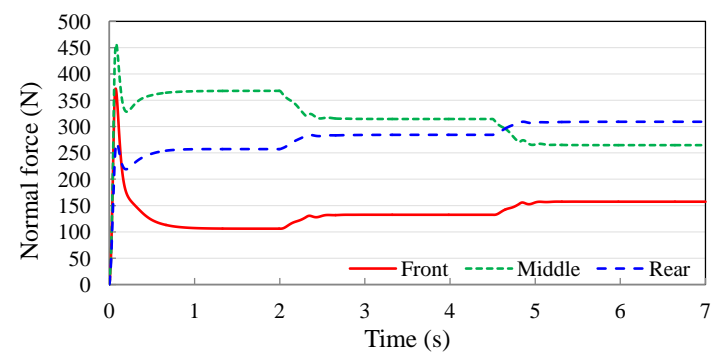

(c) Normal forces obtained in simulation

Figure 16: Effect of redundant internal actuation during drawbar-pull tests

In another set of experiments the load distribution was modified online during each test. In these experiments the RCP traveled on a straight line on soft, sandy soil. The rover started its motion with the additional mass elements attached to the front of the rover and no redundant internal actuation applied to the bogies. The position of the mass elements was not changed during the maneuver. The angular and translational velocities of the rover were the same as in the previous experiments. The normal force readings from the starboard sensors are shown in Fig. 16(a). The results show that in the first part of the motion, which corresponds to the Front_Config without redundant internal actuation, the load was distributed unevenly, with the middle wheel carrying most of the load. The second part of the motion started at $t=120 \mathrm{~s}$, where a 16-Nm moment about the bogie joints was introduced via the pneumatic actuators. Although the rover configuration remained at Front_Config, this modification resulted in reduction of the load on the middle wheels by transferring it to the rear and front wheels. To magnify this effect the actuation was increased to $32 \mathrm{Nm}$ at $t=160 \mathrm{~s}$. As expected, this modification further balanced the load distribution among the wheels. In the experiments, the magnitude of the moment that the pneumatic actuators introduced on the bogie joint was calculated as the product of the actuator force applied on the bogie and the distance from the application point to the bogie joint.

Figure 16(b) shows the NFD of the RCP during the test. The results show that redundant 
internal actuation alone without CoM repositioning was able to reduce NFD significantly. Online adjustment of the redundant internal actuation is specially useful for rover maneuvers on terrain with variable slope angles. Data from force sensors can be used internally during the rover operation to calculate the required redundant internal actuation to obtain the desired load distribution among the wheels. A similar scenario with the same actuation forces as in the experiment was simulated with the GMDL for a shorter period of time. The time history of the normal forces obtained from the simulation is shown in Fig. 16(c). The comparison of the simulation results with those of the experimental ones in Fig. 16(a) shows that the GMDL successfully captured the effect of actuation on the force distribution of the multibody rover model.

\subsection{Effect of reconfiguration on drawbar pull}

In Section 6.1 it was reported that the CoM repositioning and redundant internal actuation have a significant effect on the normal force distribution, as verified experimentally. The final objective, however, is the improvement of the rover mobility, the ability of the rover to develop drawbar pull playing a key role here. To this end, a similar set of experiments was conducted to study the way in which drawbar pull changes with the variation of NFD. In these experiments NFD was modified by means of a combination of CoM repositioning and redundant internal actuation. The drawbar-pull time history during these tests is illustrated in Fig. 17.

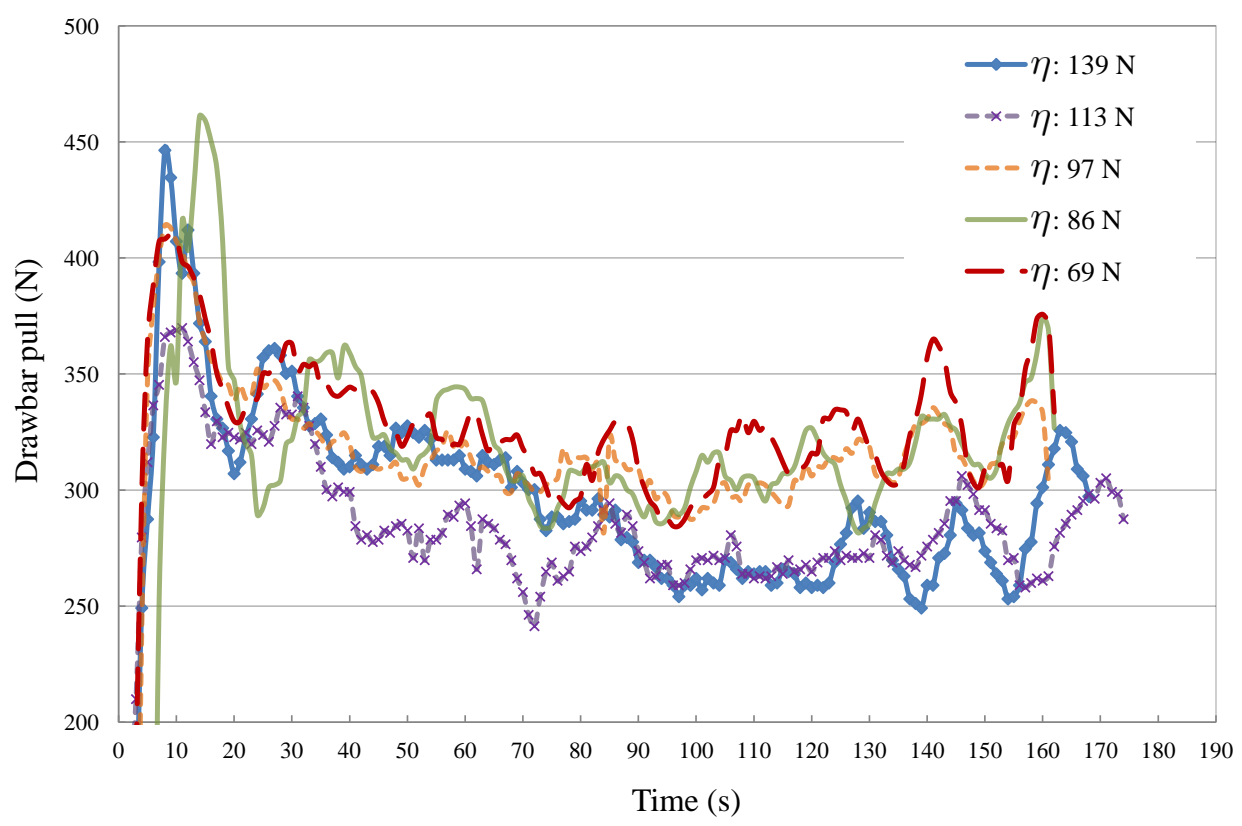

Figure 17: Drawbar pull during experiments with $60 \%$ slip and several NFD values 
Table 4: Experimental results of drawbar pull for different values of NFD (averaged for each test)

\begin{tabular}{l|lllllll}
\hline Case & 1 & 2 & 3 & 4 & 5 & 6 & 7 \\
\hline$\eta(\mathrm{N})$ & 69.0 & 82.3 & 85.9 & 97.3 & 111.3 & 113.2 & 139.4 \\
$F_{D}(\mathrm{~N})$ & 322.9 & 313.9 & 307.9 & 306.8 & 281.8 & 272.6 & 268.2 \\
\hline
\end{tabular}

Table 5: Operation conditions of the tests

\begin{tabular}{llll}
\hline $\begin{array}{l}\text { Experiment } \\
\text { No. }\end{array}$ & $\begin{array}{l}\text { Mass element } \\
\text { position }\end{array}$ & $\begin{array}{l}\text { Bogie actuation } \\
(\mathrm{Nm})\end{array}$ & $\begin{array}{l}\eta \\
(\mathrm{N})\end{array}$ \\
\hline 1 & Rear & 0 & 141 \\
2 & Front & 0 & 125 \\
3 & Front & 9 & 70 \\
4 & Front & 32 & 63 \\
\hline
\end{tabular}

For a constant ratio of $60 \%$ slip, the configurations with lower NFD provided higher drawbar pull than the ones with higher NFD. It can be shown based on the results of Section 2.1 that the relation between the NFD and the drawbar pull is also nonlinear. The average value of the drawbar pull for each test along with the value of NFD corresponding to the rover configuration in that test are reported in Table 4. Only readings from the steady-state period of the motion were considered for this.

\subsection{Comparison of experimental and simulation results}

Among the experiments reported in the previous section four cases were selected for simulation. Parameters of soil "A" from Table 1 were used in the simulation. The same angular and translational velocity specifications used in the experiments were chosen as simulation inputs. Table 5 includes details of the configuration and redundant internal actuation in the selected tests. In Experiments 3 and 4, the torque at the bogie joint was introduced halfway through the maneuver, before the system reached a steady-state motion. In the fourth test, the maximum torque was increased in two consecutive steps, first from 0 to $16 \mathrm{Nm}$, and then from 16 to 32 Nm.

Figure 18 shows the drawbar pull obtained from experimental and simulation tests. Both approaches confirm that lower values of NFD have a positive effect on the developed drawbar pull. The simulation results captured the same trends that can be appreciated in the experiments. 


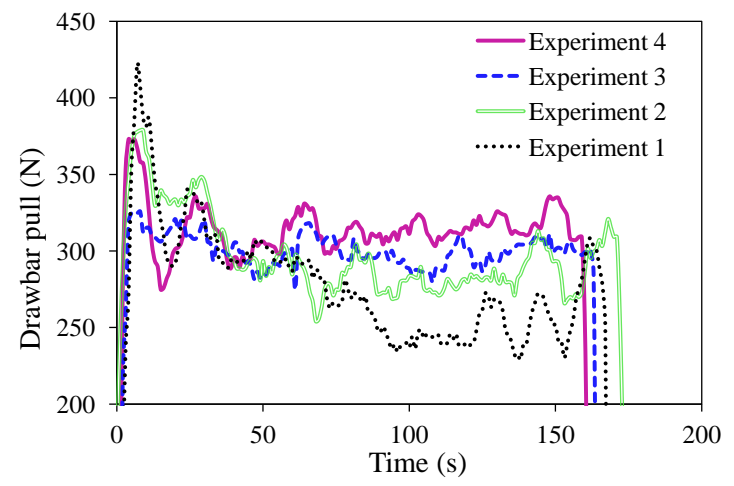

(a) Experiment

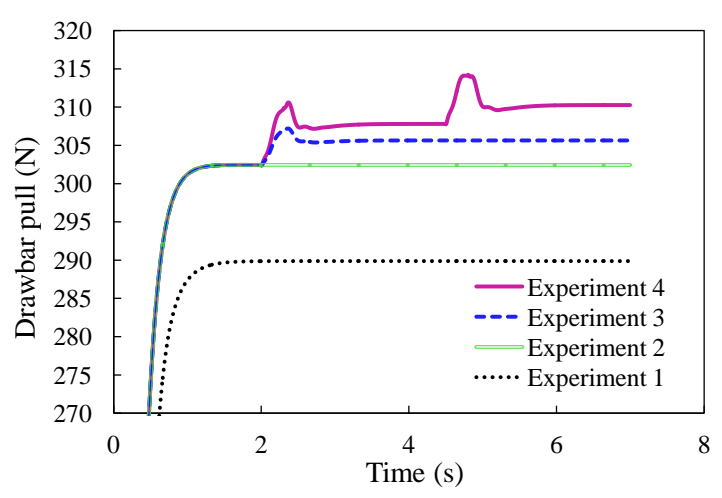

(b) Simulation

Figure 18: Experimental (a) and simulation (b) results from the drawbar-pull experiments

Comparing the plots, differences between simulation and experimental results can be observed in the average values of the drawbar pull during the steady-state part of the motion. These differences can be explained by simplifications introduced in the terramechanics relations and the modeling of the rover wheels, and also the uncertainty and variability of the terrain parameters.

\section{Effect of soil parameters and vehicle weight on the DBP-vs.-NFD relation}

The relation between the rover ability to develop drawbar pull and the NFD varies with the type of soil. The effect of modifying the NFD on the development of drawbar pull is more significant on some types of soil [14]. The frictional component of the pressure-sinkage modulus $k_{\phi}$ was found to be the most influential parameter in this regard. As discussed in Section 2.1, higher values of $k_{\phi}$ cause the $F_{D}$-vs.- $F_{n}$ plot to be closer to a straight line. Apart from the soil, vehicle weight can also influence the role of the NFD in improving rover mobility.

\subsection{Effect of the pressure - sinkage parameter $k_{\phi}$}

In order to study the effect of $k_{\phi}$ on the slope-climbing ability of the rover, a new set of simulation tests was conducted on the model of the RCP. The climbing maneuver of a slope with constant velocity was simulated. Three different slopes described in Table 6 were used. Other terrain properties were the same as in Table 1 . The slope angle for each case was selected so that the 
slip developed by the RCP with a uniform normal force distribution was the same in all cases studied, to make them comparable.

Table 6: Slope characteristics for the simulation of the climbing maneuver of the RCP

\begin{tabular}{lll}
\hline Slope & $k_{\phi}\left(\mathrm{kN} / \mathrm{m}^{n+2}\right)$ & Angle $(\mathrm{deg})$ \\
\hline 1 & 2000 & 10.0 \\
2 & 1410 & 9.3 \\
3 & 820 & 8.7 \\
\hline
\end{tabular}

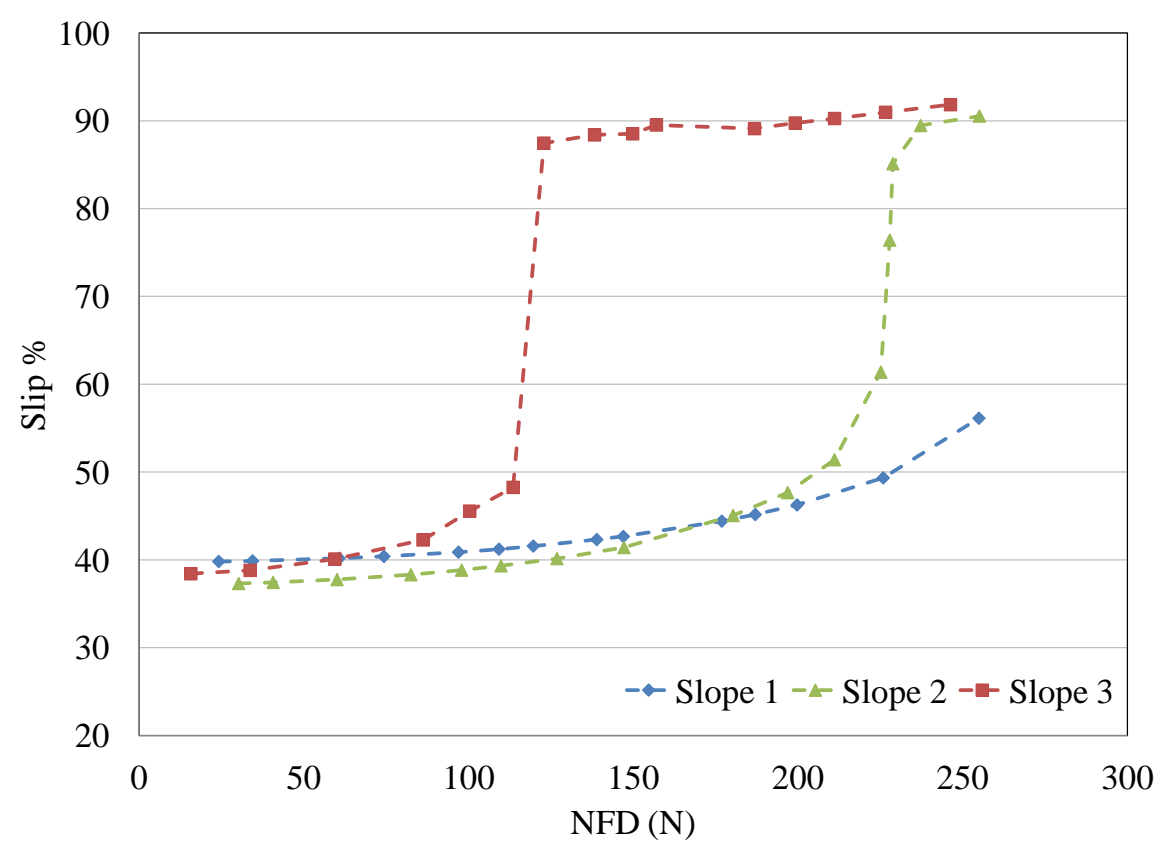

Figure 19: Slip-vs.-NFD plots for the slopes described in Table 6, with a 22.5-kg payload on the RCP

Results are recorded in Fig. 19. For small values of NFD, $\eta<100$ N, the slip remained near $40 \%$ in the three cases. However, for the terrain with the lowest $k_{\phi}$ (slope 3 ) the rover was not able to develop enough drawbar pull for $\eta>100 \mathrm{~N}$, which resulted in the slip ratio rising up to $90 \%$. A similar response was observed on slope 2 for $\eta>225 \mathrm{~N}$, while on slope 1 the slip did not exceed $60 \%$. These results suggest that the rover ability to climb a slope is more sensitive to load distribution on terrain with smaller values of $k_{\phi}$. 


\subsection{Effect of a higher normal load}

Based on simulation studies, reducing the NFD has a more significant impact on the performance of heavier rovers.

According to Fig. 2(a), regardless of the slip ratio, large normal forces accentuate the nonlinearity of the $F_{D}$-vs.- $F_{n}$ plot, displacing the operation point of the vehicle towards the right side of the $F_{D}$-vs.- $F_{n}$ plot depicted in Fig. 3 . When the rover operates in this region, making the normal force distribution more uniform will have a greater effect on the mobility of the vehicle.

To study the effect of larger loads, two sets of simulation tests with two different values of the extra weight added to the rover chassis were carried out. The selected values for the mass of the payload were $22.5 \mathrm{~kg}$ and $45 \mathrm{~kg}$. The terrain properties were the ones listed under soil "B" in Table 1. The RCP climbing a slope of $7.5^{\circ}$ with constant speed was simulated, the results being shown in Fig. 20.

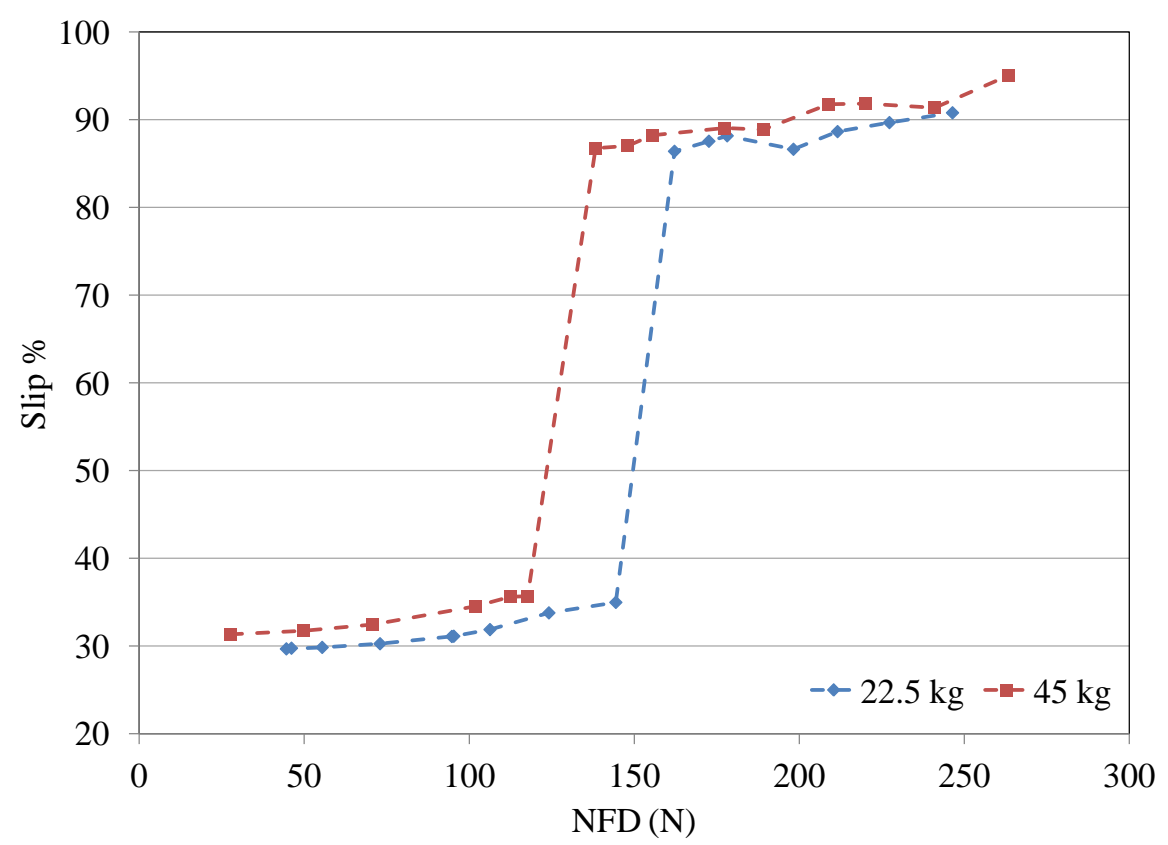

Figure 20: Slip-vs.-NFD plots for different payloads for the RCP climbing a $7.5^{\circ}$ slope on soil "B" from Table 1

The RCP showed a similar behavior in the two cases for $\eta<115 \mathrm{~N}$. When the NFD exceeded $115 \mathrm{~N}$, the rover with a 45-kg payload developed a slip close to $90 \%$, while with the lighter payload of $22.5 \mathrm{~kg}$ it was able to climb the slope keeping the slip under $40 \%$ until the NFD reached $150 \mathrm{~N}$. 


\section{Multipass effect}

The discussion in the previous sections relied on the assumptions that all the wheels had the same slip ratio and moved on the same type of soil. However, the normal force distribution can still provide relevant information about rover performance in cases in which these assumptions do not hold. In this section an example of such scenarios is discussed.

Consider a rover operation on a homogeneous soil with a straight line trajectory and the same angular velocity for all the wheels. Even in this case, the soil properties under each wheel may be different due to the multipass effect. The terrain under the front wheels can undergo significant property changes due to the compaction caused by the passage of the wheels. Therefore, the successive wheels experience a soil with different properties. In this section, the classical terramechanics models described in Section 2 were modified to consider the effect of multipass on the evaluation of the terrain reaction forces. Then, a maximization problem was formulated and solved to find the set of normal forces that resulted in the highest total drawbar pull that the rover can develop.

\subsection{Theoretical background}

The terramechanics expressions in Section 2 need to be modified to consider the effect of soil compaction by the front wheels of the vehicle. Earlier work in this area was reported by Bekker in [6] and [7], where the rolling resistance of a wheel was evaluated assuming that the pressuresinkage behavior of the soil remains the same for all the passes. Therefore, the variation of the soil reaction forces between different passes must be due to changes in the soil density or compaction. This assumption was later demonstrated experimentally [32], [26], [19].

Wong et al. [37] reported the results of pressure-sinkage bevameter tests on several terrain types. Their results suggest that the pressure-sinkage relation follows a curve like the one in Fig. 21. According to these results, first, the pressure under the wheel increases with sinkage along curve $O-A$. This curve is obtained based on the soil properties according to Eq. (1). When unloading starts at pressure $\tilde{p}_{u}$ and maximum reached sinkage $\zeta_{u}$, the pressure-sinkage relationship follows line $A$ - $B$, i.e.,

$$
\tilde{p}=\tilde{p}_{u}-k_{u}\left(\zeta_{u}-\zeta\right)
$$




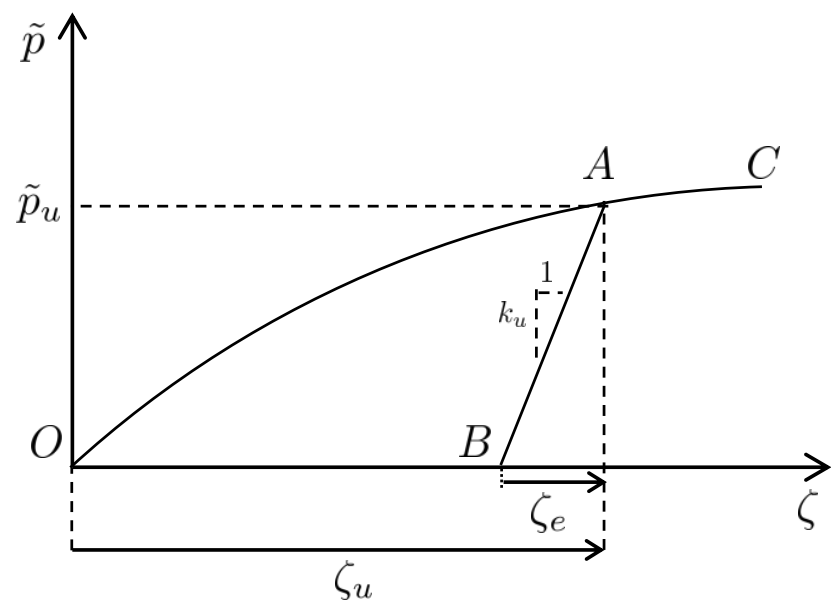

Figure 21: Schematic of repetitive loading characteristics of soil, obtained from reported bevameter tests [37]

The slope of the $A-B$ line is parameterized by $k_{u}$ which is a function of $\zeta_{u}$ :

$$
k_{u}=k_{o}+A_{u} \zeta_{u}
$$

where $k_{o}$ and $A_{u}$ have the units of $\mathrm{kN} / \mathrm{m}^{3}$ and $\mathrm{kN} / \mathrm{m}^{2}$, respectively. This relation is shown in Fig. 22. Based on Eq. (8) higher sinkage at the end of the previous loading results in higher soil compaction, which reduces the elastic rebound, $\zeta_{e}$, during unloading.

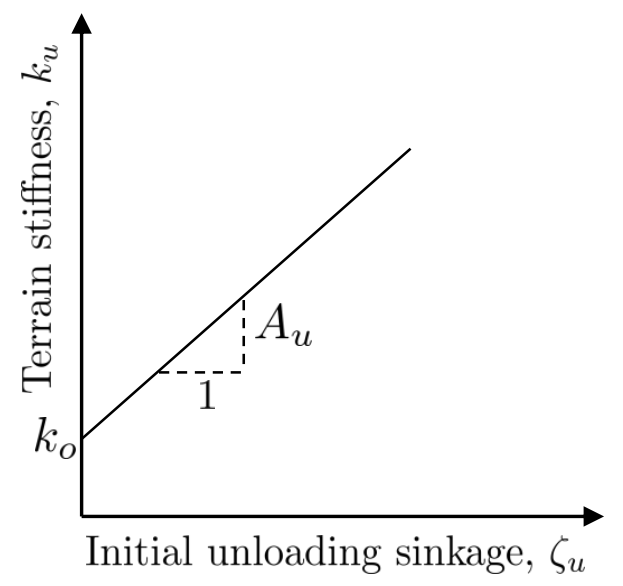

Figure 22: Schematic of soil stiffness during an unloading-reloading cycle

The soil elastic rebound takes the total sinkage back to $\zeta=\zeta_{u}-\zeta_{e}$. When the terrain is subject to the next wheel passage Eq. (7) can also represent the elastic reloading during which the sinkage can increase up to $\zeta_{u}$. If the pressure on the terrain exceeds $\tilde{p}_{u}$ the plastic deformation that occurs follows the original pressure-sinkage curve $O-A-C$.

In order to determine the terrain reactions the total sinkage has to be used in Eq. (1). The 


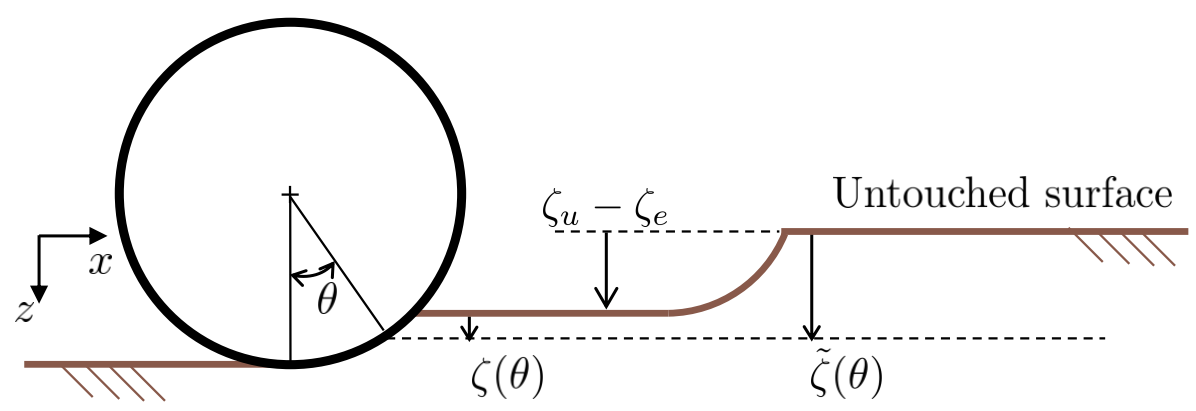

Figure 23: Schematic of wheel passage on a compacted soil and wheel local and absolute sinkage measurements

total sinkage at each point along the wheel-terrain contact area can be obtained from

$$
\tilde{\zeta}(\theta)=\zeta(\theta)+\zeta_{u}-\zeta_{e}
$$

where $\zeta(\theta)$ is the sinkage measured locally from the surface of the already compacted terrain, as shown in Fig. 23.

One complication associated with this method is that for each point on the terrain surface, the information about its deformation history, $\zeta_{u}$ and $\zeta_{e}$, as well as its hardening parameters $k_{o}$ and $A_{u}$ need to be stored [3]. Also, in the above approach the effect of wheel slip on the modification of terrain properties during each pass was not considered. An alternative approach was introduced in [19], where the variation of drawbar pull, motion resistance force and torque, slip, sinkage, and tire deflection due to multiple wheel passes was studied with an extensive set of experiments. Driven wheels were found to cause significant changes in soil properties, while towed wheels had only minor effects on them. A set of relations that fit these experimental results was reported by Senatore and Sandu in [33]. These relations can be used in simulation studies where the multipass effect needs to be considered. The approach followed in [33] is to update the soil parameters after each pass and find the terrain reactions by feeding the updated parameters to the terramechanics model. Therefore, the terramechanics model used to compute the reactions is the same for every pass. To this end, based on Holm's experimental results [19], Senatore and Sandu [33] introduced relations which give the variation of the soil properties as a function of the number of previous passes and the condition of the previous pass. For example, a relation was proposed to represent the variation of the soil density as a function of the number 
of passes $n_{p}$ and the slip ratio of the previous pass $s_{0}$, namely,

$$
\gamma_{s n}=\gamma_{s}\left[1+\left(1-e^{\frac{-s_{0}}{k_{1}}}\right) k_{2}+k_{3} n_{p}\right]
$$

where $\gamma_{s}$ is the density of the untouched soil, and $k_{1}, k_{2}$, and $k_{3}$ are dimensionless fitting constants.

Due to the lack of experimental data on the effect of multiple passes on the classical terramechanics parameters Senatore and Sandu [33] assumed that two other soil parameters have the same behavior as the soil density and the rest remain unchanged. These two parameters are $c$, soil cohesion parameter, and $K$, soil shear displacement modulus. Under the above assumption, the way these parameters change follows the model given in Eq. (10), i.e.,

$$
\begin{aligned}
& c_{s n}=c_{s}\left[1+\left(1-e^{\frac{-s_{0}}{k_{1}}}\right) k_{2}+k_{3} n_{p}\right] \\
& K_{s n}=K_{s}\left[1-\left(1-e^{\frac{-s_{0}}{k_{1}}}\right) k_{2}-k_{3} n_{p}\right]
\end{aligned}
$$

and $k_{1}, k_{2}$, and $k_{3}$ have the same value in all three equations (10), (11), and (12).

To include multipass effect in the GMDL the approach proposed in [33] was adopted. Before calculation of the terrain reactions, the soil parameters are updated based on the history of the soil. The number of previous passes and the slip ratio of the last pass are used as the input to Eqs. (10)-(12) to obtain the updated value of $\gamma, c$, and $K$.

For comparison, some simulation results reported in [33] were reproduced using the GMDL. The simulation of the motion of a single wheel on soft soil was repeated for different values of wheel slip and number of previous passes on the soil. Figure 24(a) shows the reported simulation results of the drawbar pull developed by the wheel for a range of slip ratios between 0.1 and 0.9 [33]. The same simulation tests were repeated for the second wheel pass after updating the soil properties. Information on the slip ratio of the first pass was required in order to simulate the second pass. Two cases were considered:

- Towed wheel in the first pass. A slight increase in the drawbar pull can be observed in the 


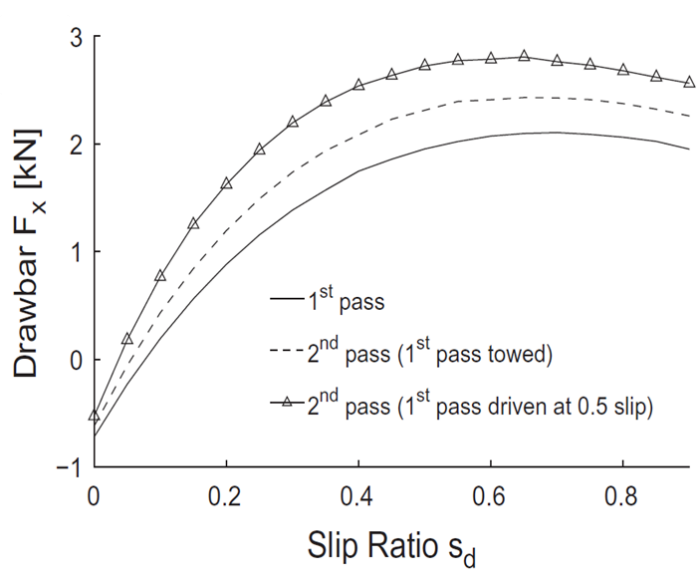

(a) Drawbar-pull variation reported in [33]

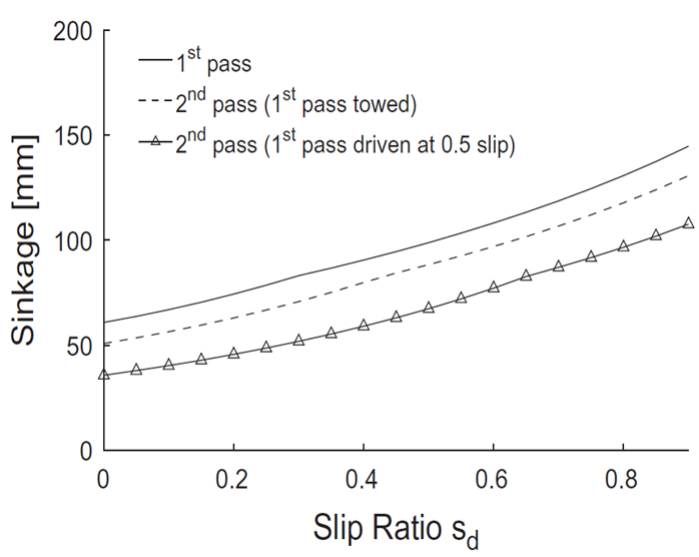

(c) Sinkage variation reported in [33]

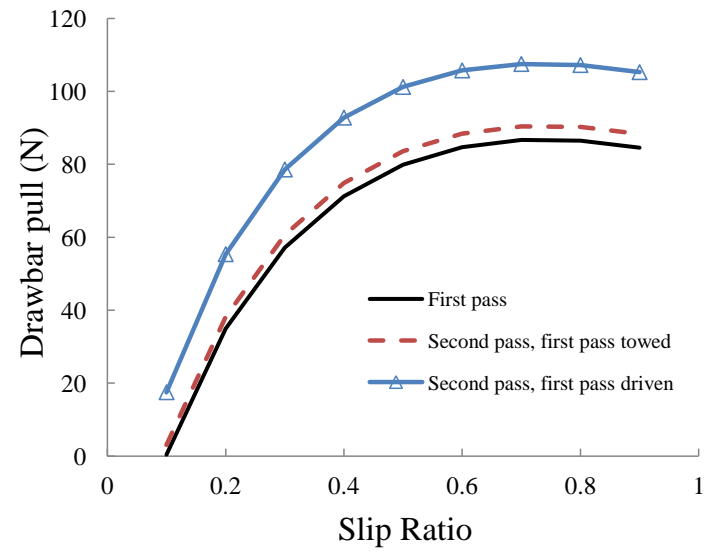

(b) Drawbar-pull variation obtained with the GMDL

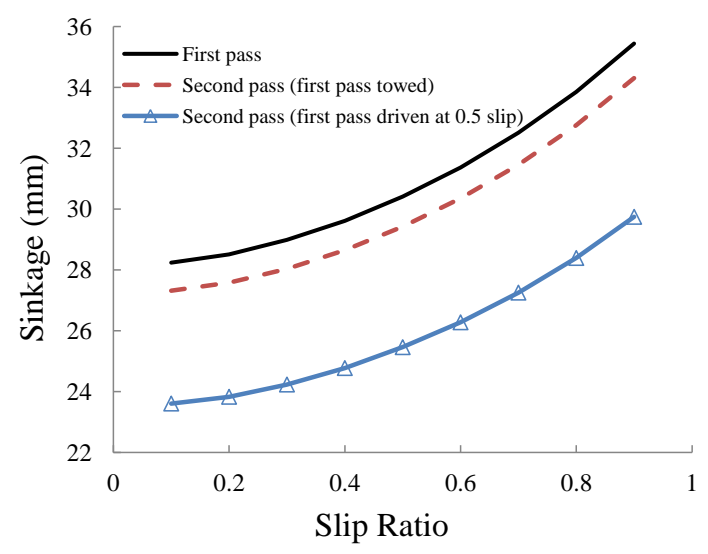

(d) Sinkage variation obtained with the GMDL

Figure 24: Simulation results of the effect of multipass

plot for this case.

- $50 \%$ slip in the first pass. Under this assumption, the soil compaction due to the first pass was considerable and, therefore, the drawbar pull generated in the second pass was higher.

In order to reproduce these results using the GMDL the soil properties and multipass parameters need to be known. The multipass parameters are given in Table 7. The soil properties introduced in [33] are in a format slightly different from the one required by our library. The advantage of this alternative format is that the dimension of the pressure-sinkage parameters is no longer dependent on the value of $n$; on the other hand, the soil properties become a function of the wheel width. In [33] the soil properties are reported in this alternative format but no information about the wheel dimensions and the vertical load supported by the wheel is provided. Due to this lack of information it was not possible to reproduce exactly the same simulation results using the GMDL. 
Table 7: Parameters defined in [33] for the multipass model

\begin{tabular}{lll}
\hline$k_{1}$ & $k_{2}$ & $k_{3}$ \\
\hline 0.1178 & 0.1672 & 0.0348 \\
\hline
\end{tabular}

Table 8: Parameters introduced in [33] for the terramechanics model

\begin{tabular}{llllll}
\hline$n$ & $c$ & $\phi$ & $k_{c}$ & $k_{\phi}$ & $K$ \\
\hline$(-)$ & $\left(\mathrm{N} / \mathrm{m}^{2}\right)$ & $(\mathrm{deg})$ & $\left(\mathrm{N} / \mathrm{m}^{n+1}\right)$ & $\left(\mathrm{kN} / \mathrm{m}^{n+2}\right)$ & $(\mathrm{m})$ \\
\hline 0.7 & 1150 & 31.1 & 22131 & 441 & 0.015 \\
\hline
\end{tabular}

The wheel width was assumed to be the same as the one of the RCP, $b=0.15 \mathrm{~m}$. The soil parameters given in [33] could then be converted to the format used in Table 8. The vertical load was set to 500 N. Figure 24(b) shows the simulation results of the drawbar pull provided by the GMDL, for the above scenarios. Comparison of Figs. 24(a) and (b) shows that modifications introduced in the soil model used in the GMDL led to prediction of the same behavior reported in [33].

Figures 24(c) and (d) illustrate the wheel sinkage values reported in [33] and the ones obtained using the GMDL for the above scenarios, respectively. With the same reasoning, the sinkage of the second pass with the previous driven wheel pass is the lowest among the three as the wheel moves on a more compacted soil. The two sets of results show a similar trend in the way sinkage changes with different wheel slip and soil history. The comparison of the simulation results obtained by the GMDL with the ones reported in [33] allows for further validation of our library.

As discussed in Section 3, the objective of this study is to find the normal force distribution among the wheels of a vehicle that results in the development of the highest possible drawbar pull. The same objective is investigated here while considering the multipass effect. In this case, the $F_{D}$-vs.- $F_{n}$ relations for different wheels of the vehicle do not follow the same curve.

The motion of a single wheel with the same dimensions as the RCP wheels, on soft soil, with $50 \%$ slip was simulated using the GMDL. Soil "B" in Table 1 was used in this simulation. Due to the smaller value of $k_{\phi}$, soil "B" undergoes a more significant compaction under the same load compared to soil "A", which makes it more suitable for the study of the multipass effect. The multipass parameters used for this simulation are listed in Table 7. Parameters $n, c, \phi, k_{c}$, $k_{\phi}$, and $K$ are used in the terramechanics model, while $k_{1}, k_{2}$, and $k_{3}$ are multipass parameters 
used in Eqs. (10)-(12).

Simulation results show that the passage of each wheel further compacts the soil; as a result, in the subsequent passages the wheels will experience lower sinkage in the compacted soil compared to the front wheels. Smaller sinkage leads to a lower rolling resistance. Also, for a given normal force and slip ratio, the drawbar pull generated on compacted soil is larger. Therefore, as can be seen in Fig. 25, for a certain value of the normal force the successive wheels develop a higher drawbar pull than the leading wheels. This behavior of the soil suggests that shifting the load toward the rear of the vehicle can increase the drawbar pull developed by the rover and improve its mobility.

\subsection{Maximization problem}

For a vehicle with $p$ wheels, the $F_{D}$-vs.- $F_{n}$ curve corresponding to wheel $i$ is generated based on the soil parameters, wheel slip, and the number and condition of previous passes on the soil. Each curve is then approximated by a polynomial that provides the value of the DBP that each wheel can develop as a function of its normal load: $F_{D i}=f_{i}\left(F_{n}\right)$. The maximization problem can be formulated as follows:

$$
\max _{\mathbf{f}_{\mathbf{n}}} \sum_{i=1}^{p} F_{D i} \quad \text { s.t. } \sum_{i=1}^{p} F_{n i}=w_{t} \quad \text { and } \quad F_{D i}>0
$$

where $\mathbf{f}_{n}=\left[F_{n 1}, F_{n 2}, \ldots, F_{n p}\right]^{\mathrm{T}}$ is the $p$-dimensional array of normal forces applied on the rover wheels, $F_{n i}$ is the normal force at the $i^{\text {th }}$ wheel, and $w_{t}$ is the total rover load, which has to be balanced by the terrain normal reactions. Next, the method developed in this section will be used to determine the optimum load distribution of the RCP for the given operation conditions.

Determining the exact load transfer that results in the optimum drawbar pull requires the solution of the above maximization problem. From Fig. 25 it can be seen that the peak for each curve occurs at a specific normal force. For example, in the case of the rear wheels associated with pass 3, if the normal load exceeds $550 \mathrm{~N}$ the developed drawbar pull decreases. This decrease is due to the fast growth of the rolling resistance force when the wheel sinkage is large. Moreover, these curves adopt a different shape for each value of the slip ratio; this difference has to be considered if the slip ratios of the wheels of the rover are not identical. The best distribution also depends on the total vertical load and rover weight. 


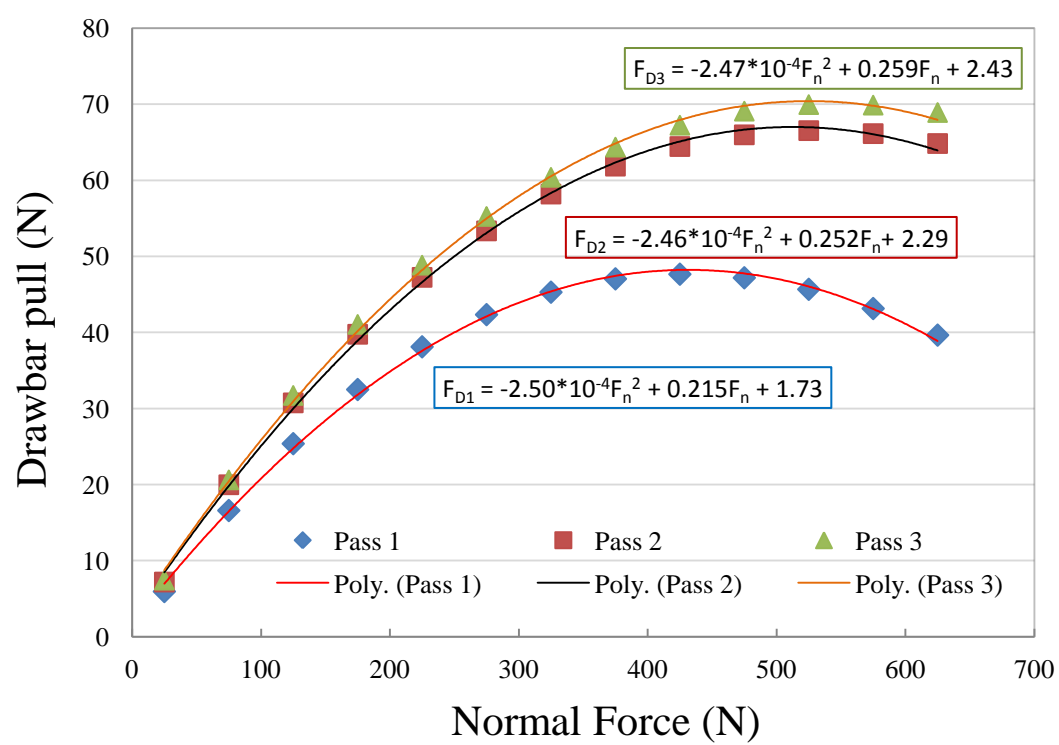

Figure 25: $F_{D}$-vs.- $F_{n}$ relation for different number of wheel passages with $50 \%$ slip on soil "B"

\subsection{Effect of normal force distribution considering the multipass effect}

The methodology introduced in Section 8.2 was employed in the simulation studies reported below. The straight line motion of the RCP on flat, sandy soil with given values of the angular velocity of the wheels and slip ratio was simulated. The same scenarios described in Section 6.3 for the drawbar-pull tests were used here. The objective of this study was to find the best normal force distribution for each test while considering the multipass effect.

In the first example the wheel slip was set to $50 \%$ and the rover operated on soil "B" of Table 1. This was the same operation condition discussed in Section 8.1 for a single wheel simulation. Therefore, the three curves illustrated in Fig. 25 can represent the $F_{D}$-vs.- $F_{n}$ curves for the front, middle, and rear wheels of the RCP. The polynomials fitted to the curves in Fig. 25, are:

$$
\begin{aligned}
& F_{D 1}=-2.50 \cdot 10^{-4} F_{n}^{2}+0.215 F_{n}+1.73 \\
& F_{D 2}=-2.46 \cdot 10^{-4} F_{n}^{2}+0.252 F_{n}+2.29 \\
& F_{D 3}=-2.47 \cdot 10^{-4} F_{n}^{2}+0.259 F_{n}+2.43
\end{aligned}
$$

Second-degree polynomials were chosen for approximating the curves as the coefficients of higher-degree terms were found to be very small for the examples studied here. The maximiza- 
Table 9: Solution of the maximization problem for the RCP operation on soil "B" with $50 \%$ slip

\begin{tabular}{l|lll}
\hline Wheel & Front $\left(F_{n 1}\right)$ & Middle $\left(F_{n 2}\right)$ & Rear $\left(F_{n 3}\right)$ \\
\hline$F_{n}(\mathrm{~N})$ & 181.5 & 259.1 & 271.8 \\
\hline
\end{tabular}

tion problem in Eq. (13) was solved subject to the constraint imposed by the total weight of the RCP, i.e.,

$$
w_{t}=2\left(F_{n 1}+F_{n 2}+F_{n 3}\right)=1424.7 \mathrm{~N}
$$

where $F_{n 1}, F_{n 2}$, and $F_{n 3}$ correspond to the normal forces on the front, middle, and rear wheels, respectively. The maximum DBP was found using a constrained gradient method. The normal forces obtained as solution resulted in $\eta=43.7 \mathrm{~N}$ and are shown in Table 9. Therefore, as these results suggest, if the multipass effect is considerable, the best load distribution is not the completely even one with $\eta=0$.

The $F_{D}$-vs.- $F_{n}$ plots in Fig. 25 were obtained by simulating the motion of a single wheel on soft soil. In order to verify the results of the optimization problem the drawbar-pull test with the RCP was simulated using the same soil and slip ratio. The simulation was repeated for a range of different normal force distributions, obtained with CoM relocation and redundant internal actuation. Figure 26(a) shows the drawbar pull developed for the different load distributions. The maximum drawbar pull occurs with the solution of the maximization problem $(\eta=43.7 \mathrm{~N})$. More uniform normal force distributions resulted in the development of smaller drawbar pulls.

The next simulation example was designed to mimic the experimental tests described in Section 6.3. The same slip ratio of $60 \%$ was prescribed for the motion of the RCP and soil "A" from Table 1 was used. The $F_{D}$-vs.- $F_{n}$ curves for the soil and slip ratio of this scenario were obtained by simulating a single wheel motion. The relations below represent the second degree polynomials that closely fit the curves:

$$
\begin{aligned}
& F_{D 1}=-1.59 \cdot 10^{-4} F_{n}^{2}+0.252 F_{n}+0.219 \\
& F_{D 2}=-1.63 \cdot 10^{-4} F_{n}^{2}+0.291 F_{n}+0.563 \\
& F_{D 3}=-1.64 \cdot 10^{-4} F_{n}^{2}+0.298 F_{n}+0.666
\end{aligned}
$$

Again, the maximization problem was formulated with the above expressions and subject to 
Table 10: Solution of the maximization problem for the RCP operation on soil "A" with $60 \%$ slip

\begin{tabular}{l|lll}
\hline Wheel & Front $\left(F_{n 1}\right)$ & Middle $\left(F_{n 2}\right)$ & Rear $\left(F_{n 3}\right)$ \\
\hline$F_{n}(\mathrm{~N})$ & 152.2 & 270.5 & 289.7 \\
\hline
\end{tabular}

the constraint in Eq. (15). The optimum normal forces in this case, which resulted in $\eta=66.6$ $\mathrm{N}$, are shown in Table 10.

Figure 26(b) shows the drawbar pull developed by the RCP in the simulation tests with $60 \%$ slip on soil "A". As in the previous example, the load distribution obtained in the maximization problem resulted in the maximum drawbar pull.

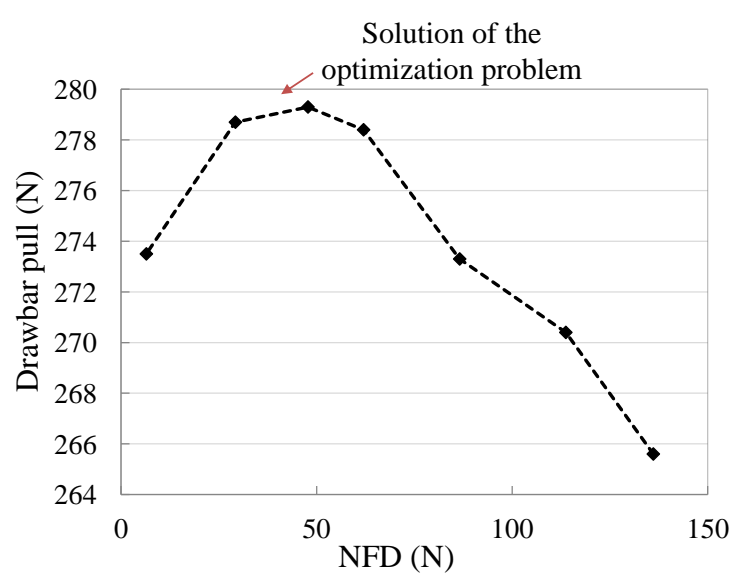

(a) Soil "B" and $50 \%$ slip

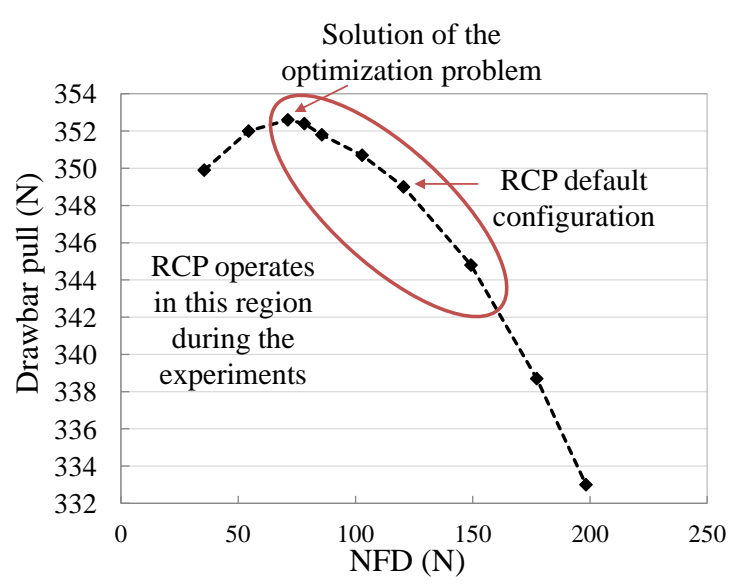

(b) Soil "A" and $60 \%$ slip

Figure 26: Drawbar-pull variation for a range of NFD considering the multipass effect.

The second scenario is intended to represent the RCP drawbar-pull experiments in the Mars Dome. Differences with respect to drawbar-pull values in Section 4 can be explained by uncertainties in the soil parameters. Despite the discrepancies in the numerical values, the trend seen in the simulation results obtained from the second case agrees with the experimental results of Section 6.2. Both simulation and experimental results suggested that in the range of $60<\eta<150 \mathrm{~N}$, lower NFD results in development of higher drawbar pull. The ellipsoid in Fig. 26(b) shows the range of the NFD covered during the experiments. The multipass effect was assumed to be negligible during the experiments and, consequently, the objective was to achieve $\eta=0 \mathrm{~N}$. Achieving a perfectly even distribution was not possible due to the limitations on the position of the CoM and maximum allowable redundant internal actuation. Therefore, experimental tests for rover configurations with $\eta<60$ could not be carried out. It is also worth mentioning that when the multipass effect is significant, the NFD indicator must be comple- 
mented with additional knowledge about the order in which the normal load is shared among the wheels. In this case, shifting the load towards the trailing wheels has a different effect on traction than loading the leading ones, even though the resultant NFD may be the same. Therefore, this information is required alongside the NFD to compare the performance of different configurations.

Comparison of the two examples discussed in this section showed that even with similar multipass parameters, due to the differences in the soil parameters and in the slip ratio, the shapes of $F_{D}$-vs.- $F_{n}$ curves were not the same. Although the rover weight was the same in the two cases, two different solutions were obtained in the maximization problems. It must be noted that due to the nonlinear shape of the curves the results obtained cannot be scaled for a different rover weight.

The scenarios discussed in this section are particular examples of conditions that lead to variations of the $F_{D}$-vs.- $F_{n}$ curves among the wheels of the rover. The methodology proposed here is suitable for other cases such as operation with different wheel slip ratios or on nonhomogeneous terrain.

\section{Conclusions}

The effect of normal force distribution on the mobility of wheeled robots was studied. A performance indicator that quantifies the dispersion of the normal force at the wheel-terrain interfaces, NFD, was defined. This indicator can be used to enhance the mobility of a wheeled robot on soft soil, including slope negotiation maneuvers, providing guidelines for configuration and actuation changes on the rover suspension in order to increase the drawbar pull. The actual impact of the normal force distribution on the available drawbar pull was found to depend on a set of factors. As a case study, the effect of the indicator on the performance of a six-wheeled rover prototype was considered. A model of the rover was built using the GMDL (Generic Multibody Dynamics Library) developed by the authors. Simulation results confirmed that reducing the NFD resulted in improved rover performance in drawbar-pull tests. These results were experimentally validated with a rover prototype. The normal load distribution was modified via CoM repositioning and redundant internal actuation. To realize the latter two pneumatic actuators were installed on the rover. The results of drawbar-pull tests with the modified rover chassis 
validated the simulation results and confirmed the effectiveness of redundant internal actuation to modify the NFD in the course of rover operations. Finally, the optimum normal force distribution was determined including the consideration of the multipass effect. Optimum load distributions were obtained as the solution of a constrained maximization problem. Simulation results confirmed that these distributions resulted in the development of the maximum possible drawbar pull. Therefore, in cases where the effect of multipass is significant, the optimum operation condition will not be the lowest possible NFD. In those cases, additional analysis similar to the one performed in this work is required.

\section{Acknowledgments}

The research work reported here was supported by the Natural Sciences and Engineering Research Council of Canada, and MDA Space Missions. This support is gratefully acknowledged. The second author would like to acknowledge the support of the Spanish Ministry of Economy through its post-doctoral research program Juan de la Cierva, contract No. JCI-2012-12376.

\section{References}

[1] Ani, O.A., Xu, H., Xue, K., gang Liu, S., yu Zhang, Z.: Analytical modeling and multiobjective optimization (MOO) of slippage for wheeled mobile robot (WMR) in rough terrain. Journal of Central South University 19(9), 2458-2467 (2012). DOI 10.1007/ s11771-012-1297-6

[2] Apostolopoulos, D.: Analytical configuration of wheeled robotic locomotion. Ph.D. thesis, Carnegie Mellon University (2001)

[3] Azimi, A.: Wheel-soil interaction modelling for rover simulation and analysis. Ph.D. thesis, Department of Mechanical Engineering, McGill University (2013)

[4] Azimi, A., Hirschkorn, M., Ghotbi, B., Kövecses, J., Angeles, J., Radziszewski, P., Teichmann, M., Courchesne, M., Gonthier, Y.: Terrain modelling in simulation-based performance evaluation of rovers. Canadian Aeronautics and Space Journal 57(1), 24-33 (2011). DOI 10.5589/q11-005 
[5] Bayo, E., Ledesma, R.: Augmented Lagrangian and mass-orthogonal projection methods for constrained multibody dynamics. Nonlinear Dynamics 9(1-2), 113-130 (1996). DOI 10.1007/BF01833296

[6] Bekker, M.G.: Off the Road Locomotion. The University of Michigan Press, Ann Arbor, Michigan (1960)

[7] Bekker, M.G.: Theory of Land Locomotion. The University of Michigan Press, Ann Arbor, Michigan (1965)

[8] Campion, G., Bastin, G., D’Andréa-Novel, B.: Structural properties and classification of kinematic and dynamic models of wheeled mobile robots. IEEE Transactions on Robotics and Automation 12(1), 47-62 (1996). DOI 10.1109/70.481750

[9] Ding, L., Deng, Z., Gao, H., Nagatani, K., Yoshida, K.: Planetary rovers' wheel-soil interaction mechanics: New challenges and applications for wheeled mobile robots. Intelligent Service Robotics 4(1), 17-38 (2011). DOI 10.1007/s11370-010-0080-5

[10] Freitas, G., Gleizer, G., Lizarralde, F., Hsu, L., Salvi dos Reis, N.R.: Kinematic reconfigurability control for an environmental mobile robot operating in the Amazon rain forest. Journal of Field Robotics 27(2), 197-216 (2010). DOI 10.1002/rob.20334

[11] Ghotbi, B.: Performance evaluation and dynamics of rovers for planetary exploration. Ph.D. thesis, McGill University (2015)

[12] Ghotbi, B., González, F., Azimi, A., Bird, W., Kövecses, J., Angeles, J., Mukherji, R.: Analysis, optimization, and testing of planetary exploration rovers: Challenges in multibody system modelling. In: Proceedings of Multibody Dynamics 2013 - ECCOMAS Thematic Conference. Zagreb, Croatia (2013)

[13] Ghotbi, B., González, F., Kövecses, J., Angeles, J.: Vehicle-terrain interaction models for analysis and performance evaluation of wheeled rovers. In: Proceedings of the 2012 IEEE/RSJ International Conference on Intelligent Robots and Systems, IROS 2012, pp. 3138-3143. Vilamoura, Portugal (2012). DOI 10.1109/IROS.2012.6386208

[14] Ghotbi, B., González, F., Kövecses, J., Angeles, J.: Effect of normal force dispersion on the mobility of wheeled robots operating on soft soil. In: Proceedings of the 2014 IEEE International Conference on Robotics and Automation, ICRA 2014, pp. 6612-6617. Hong Kong, China (2014). DOI 10.1109/ICRA.2014.6907835 
[15] Ghotbi, B., González, F., Kövecses, J., Angeles, J.: Effect of redundant actuation on the mobility of wheeled robots on unstructured terrain. In: Proceedings of the ASME 2015 International Design Engineering Technical Conferences \& Computers and Information in Engineering Conference IDTC/CIE 2015, DETC2015-47614. Boston, MA (2015)

[16] Ghotbi, B., González, F., Kövecses, J., Angeles, J.: Mobility assessment of wheeled robots operating on soft terrain. In: the 10th Conference on Field and Service Robotics (FSR 2015). Toronto, Canada (2015)

[17] Ghotbi, B., González, F., Kövecses, J., Angeles, J.: A novel concept for analysis and performance evaluation of wheeled rovers. Mechanism and Machine Theory 83, 137-151 (2015). DOI 10.1016/j.mechmachtheory.2014.08.017

[18] Grand, C., BenAmar, F., Plumet, F., Bidaud, P.: Stability and traction optimization of reconfigurable vehicles. Application to a hybrid wheel-legged robot. The International Journal of Robotics Research 23(10-11), 1041-1058 (2003). DOI 10.1177/0278364904047616

[19] Holm, I.C.: Multi-pass behaviour of pneumatic tires. Journal of Terramechanics 6(3), 4771 (1969). DOI 10.1016/0022-4898(69)90128-1

[20] Iagnemma, K., Dubowsky, S.: Traction control of wheeled robotic vehicles in rough terrain with application to planetary rovers. The International Journal of Robotics Research 23(10-11), 1029-1040 (2004). DOI 10.1177/0278364904047392

[21] Ishigami, G.: Terramechanics-based analysis and control for lunar/planetary exploration robots. Ph.D. thesis, Department of Aerospace Engineering, Tohoku University, Japan (2008)

[22] Kubota, T., Naiki, T.: Novel mobility system with active suspension for planetary surface exploration. In: Proceedings of the 2011 IEEE Aerospace Conference, AERO 2011, pp. 1 9. Big Sky (MT), USA (2011). DOI 10.1109/AERO.2011.5747271

[23] Kuroda, Y., Teshima, T., Sato, Y., Kubota, T.: Mobility performance evaluation of planetary rover with similarity model experiment. In: Proceedings of the 2004 IEEE International Conference on Robotics and Automation, ICRA 2004, vol. 2, pp. 2098-2103. New Orleans, LA, USA (2004). DOI 10.1109/ICRA.2014.6907835

[24] Lamon, P., Krebs, A., Lauria, M., Siegwart, R., Shooter, S.: Wheel torque control for a rough terrain rover. In: Proceedings of the 2004 IEEE International Conference on Robotics and 
Automation, ICRA 2004, vol. 5, pp. 4682 - 4687. New Orleans, LA, USA (2004). DOI 10.1109/ROBOT.2004.1302456

[25] Lindemann, R.A., Voorhees, C.J.: Mars exploration rover mobility assembly design, test and performance. In: Proceedings of the IEEE International Conference on Systems, Man and Cybernetics, vol. 1, pp. 450-455. Waikoloa, HI, USA (2005). DOI 10.1109/ICSMC. 2005.1571187

[26] Liston, R.A., Martin, L.A.: Multi-pass behavior of a rigid wheel. In: The 2nd International Conference of ISTVS. Quebec (1966)

[27] MacMahon, S.: Modelling and contact analysis of planetary exploration rovers. Master's thesis, McGill University (2016)

[28] Michaud, S., Richter, L., Patel, N., Thüer, T., Huelsing, T., Joudrier, L., Siegwart, R., Ellery, A.: RCET: Rover Chassis Evaluation Tools. In: Proceedings of the 8th ESA Workshop on Advanced Space Technology for Robotics and Automation (ASTRA), paper O-01. Noordwijk, The Netherlands (2004)

[29] Morales, R., Sira-Ramírez, H., Somolinos, J.A.: Robust control of underactuated wheeled mobile manipulators using GPI disturbance observers. Multibody System Dynamics 32(4), 511-533 (2014). DOI 10.1007/s11044-013-9401-8

[30] Nagatani, K., Ikeda, A., Sato, K., Yoshida, K.: Accurate estimation of drawbar pull of wheeled mobile robots traversing sandy terrain using built-in force sensor array wheel. In: Proceedings of the 2009 IEEE/RSJ International Conference on Intelligent Robots and Systems, IROS 2009, pp. 11-15. St. Louis, USA (2009). DOI 10.1109/IROS.2009.5354566

[31] Newmark, N.M.: A method of computation for structural dynamics. Journal of the Engineering Mechanics Division, ASCE 85(3), 67-94 (1959)

[32] Reece, A.R.: Problems of soil vehicle mechanics. Tech. Rep. Report No. 97, Land Locomotion Laboratory, ATAC, Warren, Michigan (1964)

[33] Senatore, C., Sandu, C.: Off-road tire modeling and the multi-pass effect for vehicle dynamics simulation. Journal of Terramechanics 48(4), 265-276 (2011). DOI 10.1016/j.jterra.2011.06.006 
[34] Thueer, T., Krebs, A., Siegwart, R., Lamon, P.: Performance comparison of rough-terrain robots - simulation and hardware. Journal of Field Robotics 24(3), 251-271 (2007). DOI 10.1002/rob.20185

[35] Thueer, T., Siegwart, R.: Mobility evaluation of wheeled all-terrain robots. Robotics and Autonomous Systems 58(5), 508-519 (2010). DOI 10.1016/j.robot.2010.01.007

[36] Wong, J.Y.: Theory of Ground Vehicles, fourth edn. John Wiley \& Sons, Inc, New Jersey (2008)

[37] Wong, J.Y., Garber, M., Preston-Thomas, J.: Theoretical prediction and experimental substantiation of the ground pressure distribution and tractive performance of tracked vehicles. Proceedings of the Institution of Mechanical Engineers, Part D: Journal of Automobile Engineering 198(4), 265-285 (1984). DOI 10.1243/PIME_PROC_1984_198_155_02

[38] Yoshida, K., Watanabe, T., Mizuno, N., Ishigami, G.: Field and Service Robotics, STAR 24, chap. Terramechanics-Based Analysis and Traction Control of a Lunar/Planetary Rover, pp. 225-234. Springer-Verlag (2006). DOI 10.1007/10991459_22 